\title{
Complete Minors, Independent Sets, and Chordal Graphs
}

\author{
József Balogh \\ University of Illinois \\ University of California, San Diego \\ jobal@math.uiuc.edu* \\ Hehui Wu \\ University of Illinois \\ hehui2@math.uiuc.edu $\ddagger$
}

May 28, 2018

\begin{abstract}
The Hadwiger number $h(G)$ of a graph $G$ is the maximum size of a complete minor of $G$. Hadwiger's Conjecture states that $h(G) \geq \chi(G)$. Since $\chi(G) \alpha(G) \geq|V(G)|$, Hadwiger's Conjecture implies that $\alpha(G) h(G) \geq|V(G)|$. We show that $\left(2 \alpha(G)-\left\lceil\log _{\tau}(\tau \alpha(G) / 2)\right\rceil\right) h(G) \geq|V(G)|$ where $\tau \approx 6.83$. For graphs with $\alpha(G) \geq 14$, this improves on a recent result of Kawarabayashi and Song who showed $(2 \alpha(G)-2) h(G) \geq|V(G)|$ when $\alpha(G) \geq 3$.
\end{abstract}

\footnotetext{
*This material is based upon work supported by NSF CAREER Grant DMS-0745185 and DMS-0600303, UIUC Campus Research Board Grants 09072 and 08086, and OTKA Grant K76099.

${ }^{\dagger}$ Work supported by 2008 REGS Program of the University of Illinois and the National Science Foundation through a fellowship funded by the grant "EMSW21-MCTP: Research Experience for Graduate Students" (NSF DMS 08-38434).

${ }^{\ddagger}$ Work supported by the National Science Foundation through a fellowship funded by the grant "EMSW21-MCTP: Research Experience for Graduate Students" (NSF DMS 08-38434).
} 


\section{Introduction}

Hadwiger's Conjecture [10] from 1943 states the following (see [18] for a survey):

Conjecture. For every $k$-chromatic graph $G, K_{k}$ is a minor of $G$.

Hadwiger's Conjecture for $k=4$ was proved by Dirac [6], the case $k=5$ was shown equivalent to the Four Color Theorem [1, 2, 16] by Wagner [19] and the case $k=6$ was shown equivalent to the Four Color Theorem by Robertson et al. 17. Hadwiger's Conjecture for $k \geq 7$ remains open. Let $h(G)$ denote the Hadwiger number, the size of the largest complete minor of $G$. Since $\alpha(G) \chi(G) \geq|V(G)|$, Hadwiger's Conjecture implies the following conjecture, which was observed in [8], 14], and [21].

Conjecture. For every graph $G, \alpha(G) h(G) \geq|V(G)|$.

This conjecture seems weaker than Hadwiger's Conjecture, however Plummer, Stiebitz, and Toft 15 showed that for graphs with $\alpha(G)=2$, the two conjectures are equivalent. In 1981, Duchet and Meyniel [8] showed that $(2 \alpha(G)-1) h(G) \geq|V(G)|$. No general improvement on this theorem has been made for the case $\alpha(G)=2$. Seymour asked for any improvement on this result for $\alpha(G)=2$, conjecturing that there exists an $\epsilon>0$ such that if $\alpha(G)=2$, then $G$ has a complete minor of size $(1 / 3+\epsilon) n$. Recently, Kawarabayashi, Plummer, and Toft [1] showed that $(4 \alpha(G)-3) h(G) \geq 2|V(G)|$ when $\alpha(G) \geq 3$ and Kawarabayashi and Song 12 improved this to $(2 \alpha(G)-2) h(G) \geq|V(G)|$ when $\alpha(G) \geq 3$. Wood [20] proved $(2 \alpha(G)-1)(2 h(G)-5) \geq 2|V(G)|-5$ for all graphs $G$. Our main result is to improve the bound for graphs with $\alpha(G) \geq 14$.

Theorem 1. Let $G$ be an n-vertex graph. Then $K_{\lceil n / r\rceil}$ is a minor of $G$, where

$$
r=2 \alpha(G)-\left\lceil\log _{\tau}(\tau \alpha(G) / 2)\right\rceil \text { and } \tau=\frac{2 \sqrt{2}}{\sqrt{2}-1} \approx 6.83 .
$$

Using a more careful analysis, we are able to improve the result for $\alpha(G)=5$.

Theorem 2. Let $G$ be an n-vertex graph with $\alpha(G)=5$. Then $K_{5 n / 38}$ is a minor of $G$.

The proof of Theorem 2 appears in the appendix which is posted online [3].

A graph $G$ is perfect if $\chi(H)=\omega(H)$ for every induced subgraph $H$ of $G$. For two vertex sets $T, S \subseteq$ $V(G)$, we say $T$ touches $S$ if $T \cap S \neq \emptyset$ or there is an edge $x y \in E(G)$ with $x \in T$ and $y \in S$. For $T \subseteq V(G)$, we define $\alpha(T)=\alpha(G[T])$ and $N(T)=\{x \in V(G): \exists y \in T, x y \in E(G)\}=\cup_{v \in T} N(v)$. If $H$ is a subgraph of $G$ and $T \subseteq V(G)$, then we define $H \cap T=G[V(H) \cap T]$. $H$ is a spanning subgraph of $G$ if $H$ is a subgraph of $G$ and $V(H)=V(G)$. A graph $G$ is chordal if $G$ has no induced cycle of length at least 4 . A vertex is simplicial if its neighborhood is a clique. A simplicial elimination ordering is an order $v_{n}, \ldots, v_{1}$ in which vertices can be deleted so that each vertex $v_{i}$ is a simplicial vertex of the graph induced by $\left\{v_{1}, \ldots, v_{i}\right\}$. A partial simplicial elimination ordering is an ordered vertex set $U=\left\{v_{1}, \ldots, v_{k}\right\} \subseteq V(G)$, such that for each $v_{i} v_{j} \notin E(G)$ with $i<j$ and $v_{i}, v_{j} \in U$ and each component $C$ of $G-\left\{v_{1}, \ldots, v_{j}\right\}$, at most one of $v_{i}$ or $v_{j}$ touches $C$. Dirac [7] proved that a graph is chordal if and only if it has a simplicial elimination ordering, and Berge [4] observed that by greedily coloring the vertices of a simplicial elimination ordering one obtains an $\omega(G)$-coloring of $G$, proving that chordal graphs are perfect.

Let $f: V(G) \rightarrow \mathbb{Q}^{+}$be a weight function on $V(G)$. For $A \subseteq V(G)$, define $f(A)=\sum_{v \in A} f(v)$. Then the weighted independence number of $G$ relative to $f$ is

$$
\alpha_{f}(G)=\max \{f(A): A \text { is an independent set in } G\} .
$$

We shall need the following result.

Theorem 3. Let $H$ be a perfect graph and $f$ a weight function on $V(H)$. Then

$$
\omega(H) \geq\left\lceil\frac{f(V(H))}{\alpha_{f}(H)}\right\rceil .
$$


The goal of our algorithm is to find a minor $H$ of $G$ such that $H$ is a chordal graph, and then to devise a weight function on the vertices of $H$ to which we apply Theorem 3 , Most of the time, the weight of a vertex $v$ in $H$ is the number of vertices of $G$ which are contracted to $v$. The algorithm builds the minor $H$ by using two operations: extension and breaking. The key property is that at each step, the algorithm uses the operations to increase the number of vertices in a partial simplicial elimination ordering. Once all vertices are included in the partial simplicial elimination ordering, we have a simplicial elimination ordering, so that the algorithm has produced a chordal graph.

In Section 2.2, we provide an algorithm which yields an alternate proof of Kawarabayashi and Song's [12] result.

Theorem 4. Let $G$ be an n-vertex graph. Then $K_{\lceil r\rceil}$ is a minor of $G$, where

$$
r= \begin{cases}n & \alpha(G)=1, \\ \frac{n}{3} & \alpha(G)=2, \\ \frac{n}{2 \alpha(G)-2} & \alpha(G) \geq 3 .\end{cases}
$$

We say that $G$ has an odd complete minor of order at least $\ell$ if there are $\ell$ disjoint trees in $G$ such that every two of them are joined by an edge, and in addition, all the vertices of the trees are two-colored in such a way that edges within trees are bichromatic and edges between trees are monochromatic. Gerards and Seymour conjectured that if a graph has no odd complete minor of order $\ell$, then it is $(\ell-1)$-colorable. This is substantially stronger than Hadwiger's Conjecture. The algorithm used by Duchet and Meyniel to prove $(2 \alpha(G)-1) h(G) \geq|V(G)|$ produces odd complete minors. The algorithm in our alternate proof of Kawarabayashi and Song's [12] result in Section 2.2 can be shown to produce an odd complete minor, so every graph with $\alpha(G) \geq 3$ has an odd complete minor of size at least $n /(2 \alpha(G)-2)$. With a little more work, we can show that our algorithm in Section 2.3 not only produces a complete minor but actually produces an odd complete minor. Therefore every graph $G$ has an odd complete minor of size at least $n /\left(2 \alpha(G)-\log _{\tau}(\tau \alpha(G) / 2)\right)$.

The rest of the paper is organized as follows: in Section 2 we define the operations and define the algorithms, in Section 3 we prove some lemmas and theorems about the operations used during the algorithms, and in Section 4 we analyze the algorithm. In the appendix posted online [3] we specialize the algorithm to $\alpha(G)=5$, and by changing the weight function we find a complete minor of size at least $5 n / 38$, which is slightly larger than the $n / 8=5 n / 40$ produced by the general algorithm.

After completing our work, we learned that Fox [9] proved using claw-free structural theorems of Chudnovsky and Fradkin [5] that every graph $G$ has a complete minor of size at least $\frac{|V(G)|}{(2-c) \alpha(G)}$ where $c \approx 0.017$. Our result is better when $\alpha(G) \leq 230$ and has the advantage of producing an odd complete minor.

\section{Definition of the algorithms}

The algorithm first builds a family of disjoint vertex sets spanning connected graphs which partition $V(G)$ and a spanning subgraph of $G$. We start with the empty family and at each step apply an operation which either adds a new set to the family, adds vertices to an existing set in the family, or updates the spanning subgraph. To identify the spanning subgraph, we color the edges of $G$ : initially all edges are blue and during the algorithm we color some edges red. We denote the spanning subgraph induced on the blue edges by $G_{b}$. When we color some edges red, we make sure that each element in the family spans a connected graph in $G_{b}$. Once we have obtained a partition of $V(G)$, we define a graph $H$ by starting with $G_{b}$ and contracting each set of the partition to a single vertex. We need the spanning subgraph $G_{b}$ because starting from $G$ and contracting each set in the partition might not yield a chordal graph. Throughout this paper, a subscript of $G$ is implied on $\alpha$ and $N$. 


\subsection{Operations used in the algorithm}

There are two operations that are carried out by the algorithm: extending and breaking. We are given a labeled (ordered) family of disjoint vertex sets $\mathcal{F}$ and a red/blue coloring of the edges of $G$. Let $U=$ $V(G)-\cup_{T \in \mathcal{F}} T$, and let $G_{b}$ be the spanning subgraph of blue edges. We define the following operations:

Extending $T$ into $X$ by $k$ : Let $T \in \mathcal{F}$, let $X \subseteq U$ such that $G_{b}[X \cup T]$ is connected, there are no red edges between $T$ and $X$, and let $k \in \mathbb{Z}^{+}$such that $k \leq \alpha(X-N(T))$. The operation extends $T$ into $X$ by $k$ by adding at most $2 k$ vertices from $X$ into $T$ so that the new $G_{b}[T]$ is still connected and we increase $\alpha(T)$ by at least $k$. When extending $T$ into $X$, the order of the sets in $\mathcal{F}$ is unchanged. In the extension we always follow the algorithm described in the proof of Lemma 12, which shows that such an extension is possible. Extending $T$ into $X$ by $k$ is always acceptable.

Breaking $X$ by $k$ : Let $k$ be a positive integer, and let $X \subseteq U$ such that $X$ does not touch $U-X$ in $G$ (i.e. $X$ is a union of some components of $G[U]$ ).

Step (a): For any $T \in \mathcal{F}$ and any component $D$ of $G[X]$ with $\alpha(D-N(T))=\alpha(D)$, we color all edges between $T$ and $D$ red.

Step (b): If there exists a component $D$ of $G[X]$ with independence number at least $k$, let $I$ be an independent set in $D$ with $|I| \geq k$ and let $v$ be any vertex in $I$. Add $T=\{v\}$ to $\mathcal{F}$ and then extend $T$ into $D-T$ by $k-1$. Lemma 13 shows that $T, D-T, k$ satisfy the conditions in the extension. We then set $X:=X-T$ and continue Step (b) until every component in $G[X]$ has independence number strictly less than $k$. The new sets produced are added last in the ordering of $\mathcal{F}$.

Definition. We say that breaking $X$ by $k$ is acceptable if both of the following conditions hold before we start breaking (before Step (a)):

- For all $T \in \mathcal{F}$ and every component $D$ of $G[X]$ either the edges between $T$ and $D$ are already red, or $\alpha(D-N(T))=\alpha(D)$ (the edges will become red in Step (a)), or $\alpha(D-N(T))<k$.

- For every component $D$ of $G[X], \alpha(D)<2 k$.

In other words, an acceptable breaking means each set $T$ in the original family and each component $D$ of $U$ will either have the edges between $T$ and $D$ colored red or touch with blue edges every set born during Step (b) in $D$, and the new sets will touch each other as well.

Definition. We say that $\mathcal{F}$ is formed by acceptable operations in $G$ if $\mathcal{F}$ is formed by starting with the empty family and then performing any sequence of acceptable operations. When we extend $T$ into $X$ by $k$ we say that the amount of the extension is $k$. For $T \in \mathcal{F}$, define $\operatorname{ext}(T)$ to be one plus the total amount of extensions of $T$, which includes the extensions in the breaking when $T$ was born and all other extensions of $T$.

In Theorem [5] we show that we obtain a chordal graph when we start with the graph $G_{b}$ and contract each set of the partition.

Theorem 5. Let $\mathcal{F}$ be a partition of $V(G)$ formed by acceptable operations in $G$, and let $G_{b}$ be the spanning subgraph of blue edges. Let $H$ be the graph obtained by starting from $G_{b}$ and contracting each set of $\mathcal{F}$ to a single vertex. Then $H$ is a chordal graph.

Lemma 6. Consider a family $\mathcal{F}$ formed by a sequence of operations in $G$. Then for every $T \in \mathcal{F},|T| \leq$ $2 \operatorname{ext}(T)-1$. Also, $\operatorname{ext}(T) \leq \alpha(T)$.

An acceptable breaking of $X$ by $k$ requires that for each component $D$ of $G[X]$ and each $T \in \mathcal{F}$ we have $\alpha(D-N(T))$ in the correct range. The following lemma shows that we can control $\alpha(D-N(T))$ by using the extension operation.

Lemma 7. Let $T^{\prime}$ be the set formed by extending $T$ into $X$ by $k$. Then $\alpha(X-N(T))-k \geq \alpha\left(X-T^{\prime}-N\left(T^{\prime}\right)\right)$. That is, extending $T$ into $X$ by $k$ using the procedure in Lemma 12 reduces $\alpha(X-N(T))$ by at least $k$. 


\subsection{The $2 \alpha(G)-2$ algorithm}

Let $n=|V(G)|$. We are going to build a partition $\mathcal{F}$ of $V(G)$ using only a sequence of breaking operations. At any stage of the algorithm, let $U=V(G)-\cup_{T \in \mathcal{F}} T$.

Case $\alpha(G)=1$ : Note that this conclusion is obvious but we give a detailed argument to make the reader more familiar with the definitions. The algorithm is to break $V(G)$ by 1 . This is an acceptable operation because before the breaking $\mathcal{F}$ is empty and every component of $G$ has independence number 1 . Breaking $V(G)$ by 1 does not color any edges red because the family before the breaking is empty, and so the breaking results in a family of singleton sets $\mathcal{F}=\{\{v\}: v \in V(G)\}$ with $G_{b}=G$. Theorem 5 shows $G$ is chordal, and using the weight function $f(v)=1$ we have that the total weight is $n$ and $\alpha_{f}(G)=\alpha(G)=1$. Thus Theorem 3 shows that $\omega(G) \geq n$.

Case $\alpha(G)=2$ : We first break $V(G)$ by 2. This is acceptable because before the breaking $\mathcal{F}$ is empty and every component of $G$ has independence number at most 2. No edges are colored red, and so this breaking results in a family $\mathcal{F}$ of disjoint induced $P_{3} \mathrm{~s}\left(P_{3}\right.$ is the unique connected graph on three vertices with independence number 2). This family $\mathcal{F}$ is maximal because the remaining vertices (the set $U$ ) induce a disjoint union of cliques. We next break $U$ by 1 . This is acceptable because each $T \in \mathcal{F}$ dominates $U$ so $\alpha(U-N(T))=0$, and each component of $G[U]$ is a clique. Also, no edge is colored red because each $P_{3}$ in $\mathcal{F}$ dominates $U$. Thus the two breaking operations produce a partition of $V(G)$ into a maximal family of induced $P_{3}$ s and singleton sets of the remaining vertices, with all edges colored blue $\left(G_{b}=G\right)$. We now contract each $P_{3}$ to form the graph $H$, and use the weight function $f(v)=3$ for a vertex $v$ obtained by contracting a $P_{3}$, and $f(v)=1$ otherwise. Thus $f(v)$ records the number of vertices in the set in $\mathcal{F}$ that is contracted down to $v$, and the total weight $f(V(H))=n$. Theorem [5 shows that $H$ is a chordal graph. To compute $\alpha_{f}(H)$, take any independent set $I$ in $H$. This independent set corresponds to a pairwise nontouching subfamily $\mathcal{I}$ of $\mathcal{F}$. Since no edges are colored red, $\mathcal{I}$ is pairwise non-touching in $G$. Then either $\mathcal{I}$ contains one $P_{3}$ and nothing else (in which case $f(I)=3$ ) or at most two single vertices (in which case $f(I)=2$ ). Thus $\alpha_{f}(H) \leq 3$ so Theorem 3 shows that $\omega(H) \geq\lceil n / 3\rceil$, that is we have a complete minor of $G$ of size at least $\lceil n / 3\rceil$.

Case $\alpha(G) \geq 3$ : Initially, $U=V(G)$ and $\mathcal{F}=\emptyset$.

- Step 1: Let $C$ be any component of $G[U]$. If $\alpha(C)$ is 1 or 2 , then we break $C$ like in the above two cases. If $\alpha(C) \geq 3$, then we break $C$ by $\alpha(C)-1$.

- Step 2: We now update $U:=U-\cup_{T \in \mathcal{F}} T$ and continue Step 1 with a new $C$ until $U=\emptyset$.

First, all the breakings are acceptable. Consider a component $C$ we are about to break in Step 1 . Now consider any set $T$ that has already been produced, say $T$ was born when $C^{\prime}$ was broke. If $C$ is not contained in $C^{\prime}$ then there are no edges between $T$ and $C$ so $\alpha(C-N(T))=\alpha(C)$. If $C \subseteq C^{\prime}$, then $\alpha(T)=\alpha\left(C^{\prime}\right)-1$ so that $\alpha\left(C^{\prime}-N(T)\right) \leq 1$ which implies that $\alpha(C-N(T)) \leq 1$. Thus breaking $C$ by $\alpha(C)-1$ is acceptable. Because $\alpha(C-N(T)) \leq 1$, the only possibility for edges to be colored red in Step 1 is when we choose a component $C$ with $\alpha(C)=1$. Thus for each $T \in \mathcal{F}$, we have $G[T]=G_{b}[T]$.

Now consider the graph $H$ formed by starting with $G_{b}$ and contracting each set of $\mathcal{F}$. Consider the weight function $f$ on $V(H)$ where we assign to each vertex of $H$ the size of the set of $\mathcal{F}$ which it came from. Thus the total weight of $f$ on $H$ is $n$. By Theorem 5 we know that $H$ is a chordal graph.

Next, we show that $\alpha_{f}(H) \leq 2 \alpha(G)-2$. Consider any independent set $I$ in $H$. This corresponds to a pairwise non-touching (in $G_{b}$ ) subfamily $\mathcal{I}$ of $\mathcal{F}$. By Lemma $6,|T| \leq 2 \operatorname{ext}(T)-1$ so that we can bound the total weight of $I$ as follows:

$$
f(I)=\sum_{T \in \mathcal{I}}|T| \leq 2 \sum_{T \in \mathcal{I}} \operatorname{ext}(T)-|I| .
$$

If $|I|=1$ then the largest breaking we ever do is by $\alpha(G)-1$ which produce sets with $\operatorname{ext}(T) \leq \alpha(G)-1$ which have size at most $2 \alpha(G)-3$. So assume $|\mathcal{I}| \geq 2$. 
Claim 8. For any pairwise non-touching family $\mathcal{I}$ in $G_{b}, \sum_{T \in \mathcal{I}} \operatorname{ext}(T) \leq \alpha(G)$.

Proof. Define $\mu(\mathcal{I})$ to be the total number of red edges between sets of $\mathcal{I}$. Assume we have a counterexample to Lemma 8 where $\mu(\mathcal{I})$ is minimized. In other words, a pairwise non-touching family $\mathcal{I}$ in $G_{b}$ where $\sum_{T \in \mathcal{I}} \operatorname{ext}(T)>\alpha(G)$ and $\mu(\mathcal{I})$ is minimized. If $\mu(\mathcal{I})=0$, then $\mathcal{I}$ is a pairwise non-touching family in $G$ so that $\sum_{T \in \mathcal{I}} \alpha(T) \leq \alpha(G)$. By Lemma 6, $\operatorname{ext}(T) \leq \alpha(T)$ so $\sum_{T \in \mathcal{I}} \operatorname{ext}(T) \leq \alpha(G)$.

Assume now that $\mu(\mathcal{I}) \geq 1$ and take some $T, R \in \mathcal{I}$ where there is a red edge between $T$ and $R$. We will produce a subfamily $\mathcal{I}^{\prime}$ spanning fewer red edges. For edges to be colored red one of $T$ or $R$ must be a single vertex. Assume $|R|=1$, and let $C$ be the component containing $R$ (with $\alpha(C)=1$ ) chosen in Step 1 which caused the edges between $T$ and $R$ to be colored red. Thus $\alpha(C-N(T))=1$ so there exists a vertex $v \in V(C)-N(T)$. Let $\mathcal{I}^{\prime}=\mathcal{I}-R+\{v\}$. Note that since $v \in V(C)$ and $\alpha(C)=1,\{v\} \in \mathcal{F}$. We now show that $v$ does not touch any other set in $\mathcal{I}^{\prime}$. Say that there exists an $S \in \mathcal{I} \cap \mathcal{I}^{\prime}$ where $S$ touches $v$ in $G$. Since $v \in V(C)-N(T)$, we must have $T \neq S$. First assume $\operatorname{ext}(S)=1$, so that $S=\{s\}$ for some vertex $s$. Then since $s$ touches $v$ we must have $s \in V(C)$. Note that when singletons are born, their component must be a clique. But then $s$ touches $R$ using a blue edge, contradicting that $S \in \mathcal{I}$. So we can assume $\operatorname{ext}(S) \geq 2$.

First assume $T$ is indexed lower than $S$, and let $C^{\prime}$ be the component chosen in Step 1 when $T$ was born. Then $\alpha(T) \geq \operatorname{ext}(T)$ and $\operatorname{ext}(T)$ is one plus the number of extensions during the breaking so ext $(T)=$ $\alpha\left(C^{\prime}\right)-1$. Since $S$ touches $v$ and $T$ is connected to $v$ by a path of length 2 using a vertex of $C$ we have that $S$ is contained inside $C^{\prime}$. Since $\alpha(S) \geq \operatorname{ext}(S) \geq 2$ we must have $T$ touching $S$ using blue edges, contradicting that both are in $\mathcal{I}$. Now assume that $S$ is indexed lower than $T$, and let $C^{\prime}$ be the component chosen in Step 1 when $S$ was born. Then $\alpha(S) \geq \operatorname{ext}(S)=\alpha\left(C^{\prime}\right)-1$ and since $S$ touches $v$ and $T$ is connected to $v$ by a path of length 2 using a vertex of $C$ we have that $T$ is contained inside $C^{\prime}$. Since $\alpha(T) \geq \operatorname{ext}(T) \geq 2$ we have that $S$ touches $T$ using blue edges, contradicting that both are in $\mathcal{I}$. Thus $v$ does not touch any other set in $\mathcal{I}^{\prime}$, so $\mathcal{I}^{\prime}$ is pairwise non-touching in $G_{b}$ and we have reduced the number of red edges. Also, $\sum_{T \in \mathcal{I}^{\prime}} \operatorname{ext}(T)=\sum_{T \in \mathcal{I}} \operatorname{ext}(T)>\alpha(G)$ contradicting that $\mathcal{I}$ was a minimum counterexample.

Using Claim 8, we can immediately complete the proof since then $f(I) \leq 2 \alpha(G)-|I| \leq 2 \alpha(G)-2$. To summarize, we can find a complete minor of $G$ of size $\lceil r\rceil$, where $r$ is defined as in Theorem 4 .

\subsection{The $\left.2 \alpha(G)-\log _{\tau}(\tau \alpha(G) / 2)\right)$ algorithm}

Given a graph $G$, we use the operations of breaking and extending to produce a partition $\mathcal{F}$ of $V(G)$ and a spanning subgraph $G_{b}$ of blue edges. When we start the algorithm, $\mathcal{F}$ will be the empty family and $G_{b}=G$. The improvement from $2 \alpha(G)-2$ to $2 \alpha(G)-\log _{\tau}(\tau \alpha(G) / 2)$ comes from breaking each component $C$ by $\lceil(\alpha(C)+1) / 2\rceil$ so we produce sets of size approximately $\alpha(C)$, and then we extend the sets of $\mathcal{F}$ before future breakings only if it would prevent the breaking from being acceptable.

Given a graph $G$, set $G_{b}=G$ so all edges are colored blue and set $\mathcal{F}=\emptyset$.

We pick $C$ to be any component of $G\left[V(G)-\cup_{T \in \mathcal{F}} T\right]$. If $\alpha(C)=1$, we break $C$ by 1 which constitutes Step $C$. So assume $\alpha(C) \geq 2$, and run the following substeps inside $C$, which constitutes Step $C$.

- Substep 1: For each $T \in \mathcal{F}$ with $\alpha(C-N(T))=\alpha(C)$, color all edges between $T$ and $C$ red. Then let $b=\left\lceil\frac{\alpha(C)+1}{2}\right\rceil$, and let $A=V(C)$. Partition $\mathcal{F}$ into three classes.

$-\mathcal{H}_{0}=\{T \in \mathcal{F}:$ all edges between $T$ and $C$ are colored red $\}$,

$-\mathcal{H}_{1}=\left\{T \in \mathcal{F}-\mathcal{H}_{0}: \alpha(C-N(T))<\sqrt{2}(b-1)\right\}$,

$-\mathcal{H}_{2}=\mathcal{F}-\mathcal{H}_{0}-\mathcal{H}_{1}$.

- Substep 2: For any $T \in \mathcal{H}_{1}$ and any component $D$ of $G[A]$ with $b \leq \alpha(D-N(T))<\alpha(D)$, we extend $T$ into $V(D)$ by $\alpha(D-N(T))-b+1$. We then update $A:=A-T$ and continue Substep 2 until no pair $T, D$ satisfies $b \leq \alpha(D-N(T))<\alpha(D)$. Note, for the first $T$ selected during Substep 2 we will have $D=C$ so that $T$ satisfies $b \leq \alpha(C-N(T))<\alpha(C)$ and thus is extended. 
If there exists some $T \in \mathcal{H}_{1}$ which was not extended during Substep 2 and some component $D$ of $G[A]$ such that $b \leq \alpha(D-N(T))=\alpha(D)$ then we do not continue to Substep 3, instead we finish Step $C$. Otherwise, continue to Substep 3.

- Substep 3: For any $T \in \mathcal{H}_{2}$ and any component $D$ of $G[A]$ with $b \leq \alpha(D-N(T))<\alpha(D)$, we extend $T$ into $V(D)$ by $\alpha(D-N(T))-b+1$. We then update $A:=A-T$ and continue Step 3 until no pair $T, D$ satisfies $b \leq \alpha(D-N(T))<\alpha(D)$.

- Substep 4: Break $A$ by $b$.

If $\mathcal{F}$ is not yet a partition of $V(G)$, pick a new component $C$.

In Sections 3 and 4, we prove that using this algorithm we can find a complete minor of $G$ of size $\lceil n / r\rceil$, where $r$ is defined in Theorem 1 .

\section{Analysis of the operations}

\subsection{Proofs of Theorems 3 and 5}

If $V(G)=\left\{v_{1}, \ldots, v_{n}\right\}$ and $H_{1}, \ldots, H_{n}$ are pairwise disjoint graphs, then the composition $G\left[H_{1}, \ldots, H_{n}\right]$ is the graph formed by the vertex disjoint union of $H_{1}, \ldots, H_{n}$ plus the edges $x y$ where $x \in V\left(H_{i}\right), y \in V\left(H_{j}\right)$ and $v_{i} v_{j} \in E(G)$. In 1972, Lovász [13] proved that a composition of perfect graphs is perfect.

Proof of Theorem 3. First, we modify $f$ by multiplying each weight by their common denominator so that $f: V(H) \rightarrow \mathbb{Z}^{+}$. Multiplying every weight by a constant does not change $f(V(H)) / \alpha_{f}(H)$. For $v \in$ $V(H)$, define $H_{v}=f(v) K_{1}$ to be an independent set of size $f(v)$. Then define $H^{\prime}=H\left[H_{v_{1}}, \ldots, H_{v_{n}}\right]$ as a composition of $H$. Then $f(V(H))=\left|V\left(H^{\prime}\right)\right|, \omega(H)=\omega\left(H^{\prime}\right)$, and $\alpha_{f}(H)=\alpha\left(H^{\prime}\right)$. (If $I^{\prime}$ is a maximal independent set in $H^{\prime}$, then either $H_{v} \subseteq I^{\prime}$ or $H_{v} \cap I^{\prime}=\emptyset$ because $H_{v}$ is an independent set and every vertex in $H_{v}$ has the same neighborhood.) Since $H^{\prime}$ is a perfect graph, we have

$$
\omega(H)=\omega\left(H^{\prime}\right)=\chi\left(H^{\prime}\right) \geq\left\lceil\frac{\left|V\left(H^{\prime}\right)\right|}{\alpha\left(H^{\prime}\right)}\right\rceil=\left\lceil\frac{f(V(H))}{\alpha_{f}(H)}\right\rceil .
$$

We say that $\mathcal{F}=\left\{T_{1}, \ldots, T_{k}\right\}$ is a partial simplicial elimination ordering in $G$ if for every nontouching pair $T_{i}, T_{j}$ with $i<j$ and for every component $C$ of $G-T_{1}-\ldots-T_{j}$, at most one of $T_{i}$ or $T_{j}$ touches $C$. This corresponds exactly to a partial simplicial elimination ordering in the graph obtained by contracting each set of $\mathcal{F}$. We first prove that using acceptable operations we get a partial simplicial elimination ordering in the blue subgraph.

Theorem 9. Let $\mathcal{F}_{0}$ be a partial simplicial elimination ordering in $G$, and let $\mathcal{F}$ be any family formed by starting with $\mathcal{F}_{0}$ and performing any sequence of acceptable operations. Let $G_{b}$ be the spanning subgraph of blue edges after the operations. Then $\mathcal{F}$ is a partial simplicial elimination ordering in $G_{b}$.

Proof. Let $G_{b}$ be the spanning subgraph of $G$ of the blue edges at the end of all operations. We need to prove that after every acceptable operation we have a partial simplicial elimination ordering.

Lemma 10. Let $\mathcal{F}$ be a partial simplicial elimination ordering in $G_{b}$. Let $U$ be the set of vertices $V(G)-$ $\cup_{T \in \mathcal{F}} T$, and let $X \subseteq U$ be a union of some components of $G[U]$. Let $k$ be an integer such that breaking $X$ by $k$ is an acceptable operation. Then the family obtained by breaking $X$ by $k$ is a partial simplicial elimination ordering in $G_{b}$. 
Proof. Let $\mathcal{F}=\left\{T_{1}, \ldots, T_{m}\right\}$ be the original family, and let $R_{1}, \ldots, R_{\ell}$ be the sets produced when we broke $X$ by $k$. We consider a non-touching pair in $G_{b}$, and show that the pair satisfies the condition for a partial simplicial elimination ordering. We only need to consider pairs which contain at least one $R_{j}$.

Let $T_{i}, R_{j}$ be a non-touching pair in $G_{b}$. Let $D$ be the component of $G[X]$ containing $R_{j}$ (then $D$ is also a component of $G[U]$ ). We first show that all edges between $T_{i}$ and $D$ are colored red.

First assume $T_{i}$ does not touch $R_{j}$ in $G$. Then $k=\alpha\left(R_{j}\right) \leq \alpha\left(D-N\left(T_{i}\right)\right)$ so by the condition in the definition of acceptable breaking we have $\alpha\left(D-N\left(T_{i}\right)\right)=\alpha(D)$ or all edges between $T_{i}$ and $D$ are colored red, thus after the breaking all edges between $T_{i}$ and $D$ are red.

Now assume the edges between $T_{i}$ and $R_{j}$ are red. Then we either had all edges between $T_{i}$ and $D$ red before the breaking or we colored all edges between $T_{i}$ and $D$ red during the breaking. Thus we have all the edges between $T_{i}$ and $D$ colored in red.

Let $C$ be any component of $G_{b}-T_{1}-\ldots-T_{m}-R_{1}-\ldots-R_{j}$. We want to show that at least one of $T_{i}$ or $R_{j}$ does not touch $C$ using blue edges. $C$ is either contained inside $D$ or disjoint from $D$, because $D$ is a component of $G[U]=G-T_{1}-\ldots-T_{m}$ and $C$ is a connected subgraph of $G[U]$. If $C$ is disjoint from $D$ then $R_{j}$ does not touch $C$ in $G$. If $C$ is contained inside $D$, then $T_{i}$ does not touch $C$ using blue edges because all edges between $T_{i}$ and $C$ are red.

Now consider a non-touching pair $R_{i}, R_{j}$ in $G_{b}$ with $i<j$. Assume $R_{i}$ and $R_{j}$ are contained in the same component $D$ of $G[X]$. Then since $2 k>\alpha(D)$ we must have $R_{i}$ touching $R_{j}$ in $G$ so touching in $G_{b}$ (we only color edges red which have exactly one endpoint in an existing set). Thus we must have $R_{i}$ and $R_{j}$ in different components of $G[X]$. So let $C$ be any component of $G_{b}-T_{1}-\ldots-T_{m}-R_{1}-\ldots-R_{j}$, so that $V(C) \subseteq U$. Then $C[V(C) \cap X]$ is contained inside some component of $G_{b}[X]$ so $C[V(C) \cap X]$ cannot touch both $R_{i}$ and $R_{j}$ in $G_{b}$. Since there are no edges between $X$ and $U-X$ in $G$, if $R_{i}$ has no edges to $C[V(C) \cap X]$ then $R_{i}$ has no edges to $C$ and similarly if $R_{j}$ has no edges to $C[V(C) \cap X]$. Thus at most one of $R_{i}$ or $R_{j}$ touches $C$ using blue edges.

Lemma 11. Let $\mathcal{F}$ be a partial simplicial elimination ordering in $G_{b}$. Let $X \subseteq V(G)-\cup_{T \in \mathcal{F}} T$ where $G_{b}[X]$ is connected, and let $T_{i}$ be an element of $\mathcal{F}$. Then the family obtained by extending $T_{i}$ into $X$ is still a partial simplicial elimination ordering in $G_{b}$.

Proof. Let $\mathcal{F}=\left\{T_{1}, \ldots, T_{m}\right\}$ before the extension, and let $T^{\prime}$ be the set $T_{i}$ plus the vertices added during the extension. Now consider a $T_{j} \in \mathcal{F}$ where $T_{j}$ does not touch $T^{\prime}$ in $G_{b}$, let $\ell=\max \{i, j\}$ and consider any component $C$ of $G-T_{1}-\ldots-T_{\ell}-T^{\prime}$. Let $D$ be the component of $G-T_{1}-\ldots-T_{\ell}$ which contains $C$. Because $\mathcal{F}$ is a partial simplicial elimination ordering, at least one of $T_{i}$ or $T_{j}$ does not touch $D$ using blue edges. Since $G[X]$ is connected, $X$ is either contained inside $V(D)$ or disjoint from $V(D)$. If $X$ is not contained inside $V(D)$, then $D=C$ and at least one of $T_{i}$ or $T_{j}$ does not touch $C$ using blue edges. Extension does not change this, so one of $T^{\prime}$ or $T_{j}$ does not touch $C$ using blue edges. If $X$ is contained inside $V(D)$, then $T_{i}$ touches $D$ using blue edges $\left(T_{i}\right.$ touches the new vertices in $T^{\prime}$ and we only extend using blue edges) so $T_{j}$ does not touch $D$ using blue edges so does not touch $C$ using blue edges.

Clearly, Lemma 10 and Lemma 11 imply Theorem 9.

Proof of Theorem 5. Assume that $\mathcal{F}$ is formed by acceptable operations. Initially, we have that $\mathcal{F}_{0}=\emptyset$ which is trivially a partial simplicial elimination ordering. Then by Theorem 9 , $\mathcal{F}$ is a partial simplicial elimination ordering in $G_{b}$. Let $H$ be the graph obtained from $G_{b}$ by contracting each $T_{i} \in \mathcal{F}$ into a single vertex $v_{i} \in V(H)$.

We show that $H$ is chordal by giving a simplicial elimination ordering of $H$. We order the vertices of $H$ according to the ordering of the sets of $\mathcal{F}$. For each $v_{i} \in V(H)$, define $B_{i}=N\left(v_{i}\right) \cap\left\{v_{1}, \ldots, v_{i}\right\}$. Assume we had $v_{j}, v_{k} \in B_{i}$ with $j<k<i$ where $v_{j} v_{k} \notin E(H)$. Let $D$ be the component of $G_{b}-T_{1}-\ldots-T_{k}$ which contains $T_{i}$. Then $T_{j}$ does not touch $T_{k}$ in $G_{b}$, so by the condition on partial simplicial elimination ordering one of $T_{j}$ or $T_{k}$ does not touch $D$ in $G_{b}$. This contradicts that $v_{j}, v_{k} \in B_{i}$, so $B_{i}$ spans a clique. This happens for each $i$, yielding that $H$ is a chordal graph. 


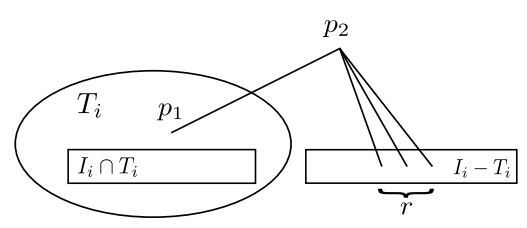

(a) Case 1

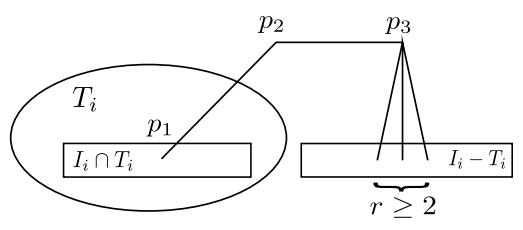

(b) Case 2

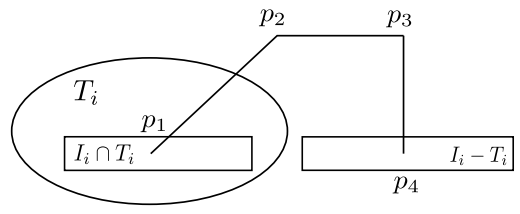

(c) Case 3

Figure 1: Extensions of $T_{i}$.

\subsection{Some properties of the operations}

In this subsection, let $G$ be any graph and $G_{b}$ any spanning subgraph of $G$. Let $T, X \subseteq V(G)$ and $k$ any integer with $T \cap X=\emptyset, G_{b}[T]$ connected, $G_{b}[X \cup T]$ connected, no red edges between $T$ and $X$, and $k \leq \alpha(X-N(T))$. (These are the conditions when we extend $T$ into $X$ by $k$ during the algorithm.)

Lemma 12. It is possible to extend $T$ into $X$ by $k$ such that $G_{b}[T]$ remains connected and $\alpha(T)$ increases by at least $k$ and $|T|$ increases by at most $2 k$.

Proof. Let $T_{0}=T$ so $T_{0}$ is the initial $T$. We use the following algorithm to produce $T_{0} \subseteq T_{1} \subseteq \ldots \subseteq T_{k}$, where $\alpha\left(T_{i}\right) \geq \alpha\left(T_{0}\right)+i$ and $\left|T_{i}\right| \leq\left|T_{0}\right|+2 i$. (Note that we do not define $T_{i}$ for every $i<k$.) Initially, let $I_{0}$ be any maximal independent set in $G\left[X-N\left(T_{0}\right)\right]$ with $\left|I_{0}\right| \geq k$.

Assume we have defined $T_{i}$ and $I_{i}$ with $i<k$. We now show how to define $T_{i+r}$ and $I_{i+r}$ for some $1 \leq r \leq k-i$.

Step 1. Choose $P$ to be a shortest path in $G_{b}\left[X \cup T_{0}\right]$ between $T_{i}$ and $I_{i}-T_{i}$. The length of $P$ is at most three because $I_{i}$ is a maximal independent set in $G_{b}\left[X-N\left(T_{0}\right)\right]$. The algorithm maintains that there are no edges between $T_{i}$ and $I_{i}-T_{i}$ when $i<k$, so the length of $P$ is at least two.

Step 2.

Case 1: Consider when $P=\left(p_{1}, p_{2}, p_{3}\right)$ with $p_{1} \in T_{i}$ and $p_{3} \in I_{i}-T_{i}$. Then we add $p_{2}$ and

$$
r=\min \left\{k-i,\left|N\left(p_{2}\right) \cap\left(I_{i}-T_{i}\right)\right|\right\} \geq 1
$$

vertices from $N\left(p_{2}\right) \cap\left(I_{i}-T_{i}\right)$ to $T_{i}$ to form $T_{i+r}$. Let $I_{i+r}=I_{i}$. Thus $\alpha\left(T_{i+r}\right)=\alpha\left(T_{i}\right)+r$ and $\left|T_{i+r}\right|=\left|T_{i}\right|+1+r$.

Case 2: Consider when $i \leq k-2$ and $P=\left(p_{1}, p_{2}, p_{3}, p_{4}\right)$ with $p_{1} \in T_{i}$ and $\left|N\left(p_{3}\right) \cap\left(I_{i}-T_{i}\right)\right| \geq 2$. Here, we add $p_{2}, p_{3}$, and

$$
r=\min \left\{k-i,\left|N\left(p_{3}\right) \cap\left(I_{i}-T_{i}\right)\right|\right\} \geq 2
$$

vertices of $N\left(p_{3}\right) \cap\left(I_{i}-T_{i}\right)$ to $T_{i}$ to form $T_{i+r}$. Let $I_{i+r} \subset G\left[X-T_{i}-N\left(T_{i}\right)\right]$ be a maximal independent set containing $I_{i}$. Then $\alpha\left(T_{i+r}\right) \geq \alpha\left(T_{i}\right)+r$ and $\left|T_{i+r}\right|=\left|T_{i}\right|+2+r$. Since $r \geq 2$, the increase in the number of vertices is at most twice the increase of $i$.

Case 3: Consider when $P=\left(p_{1}, p_{2}, p_{3}, p_{4}\right)$ with $p_{1} \in T_{i}$ and $N\left(p_{3}\right) \cap I_{i}=\left\{p_{4}\right\}$. We set $I_{i+1}=I_{i}-\left\{p_{4}\right\}+\left\{p_{3}\right\}$ and then extend $I_{i+1}$ to be a maximal independent set in $G\left[X-T_{i}-N\left(T_{i}\right)\right]$. Then $I_{i+1}$ is still a maximal independent set of size at least $k$, and we can now add $p_{2}$ and $p_{3}$ to $T_{i}$ to get $T_{i+1}$. This increases the number of vertices by two and the independence number by one.

Case 4: Consider when $i=k-1$ and $P=\left(p_{1}, p_{2}, p_{3}, p_{4}\right)$ with $p_{1} \in T_{i}$ and $\left|N\left(p_{3}\right) \cap I_{i}\right| \geq 2$. Here, we add $p_{2}$ and $p_{3}$ to $T_{i}$ to get $T_{k}$. Let $I_{k}=I_{i}-\left\{p_{4}\right\}+\left\{p_{3}\right\}$. Thus $\alpha\left(T_{k}\right) \geq \alpha\left(T_{0}\right)+k$ and $\left|T_{k}\right| \leq\left|T_{i}\right|+2$. 
Consider one step which did not produce $T_{k}$. If this step is Case 1, then we added the entire set $N\left(p_{2}\right) \cap I_{i}$ to $T_{i}$. In Case 2, we added the entire set $N\left(p_{3}\right) \cap I_{i}$ to $T_{i}$. In Case 3 , we added the entire $N\left(p_{2}\right) \cap I_{i+1}$ to $T_{i}$. In Case 4 , we always produce $T_{k}$. Note that we always maintain that there are no edges between $T_{i}$ and $I_{i}-T_{i}$ if $i<k$. If we ever added all vertices of $I_{i}$ to $T_{i}$ we would have increased $\alpha\left(T_{i}\right)$ to $\alpha\left(T_{0}\right)+k$ because $I_{i} \subseteq X-N\left(T_{0}\right)$ and $\left|I_{i}\right| \geq k$. We continue the algorithm if $i<k$ so we will eventually produce a $T_{k}$.

Lemma 13. The extension of $T=\{v\}$ into $D-T$ by $k-1$ during Step (b) of a breaking operation satisfies all the conditions of the extension.

Proof. Any edge colored red has at least one endpoint in a set of $\mathcal{F}$, so that $G_{b}[D]=G[D]$ where $G_{b}$ is the spanning subgraph of blue edges at the time of extension. Since $D$ is a component of $G[U]$, we have $G_{b}[D]$ connected. Also, since $T \subseteq V(D)$ we have no red edges between $T$ and $D-T$ at the time of extension. Finally, since we chose $v \in I$ where $I$ is an independent set of size at least $k$, we have $\alpha(D-N(v)) \geq|I-v| \geq k-1$.

Proof of Lemma 6 . The extending operation does not produce new sets, so the only way to produce a new set is by breaking some set $X$ by $k$. In Step (b) of the breaking, we initially have $|T|=1$, and then we extend $T$ by $k-1$ which adds at most $2 k-2$ new vertices, so $T$ has at most $2 k-1$ vertices. Since the independence number increased by at least $k-1$, we have $\alpha(T) \geq k$. Extending $T$ by $k$ increases the number of its vertices by at most $2 k$ and its independence number by at least $k$. Thus $|T| \leq 2 \operatorname{ext}(T)-1$ and $\alpha(T) \geq \operatorname{ext}(T)$.

Proof of Lemma 7 . Let $B=G[X-N(T)]$. Assume towards a contradiction that $\alpha(B)-k+1 \leq \alpha(X-$ $T^{\prime}-N\left(T^{\prime}\right)$ ), and let $I=I_{k}$ be the independent set used at the end of the proof of Lemma 12. Then $\alpha(B) \geq \alpha\left(B \cap T^{\prime}\right)+\alpha\left(B-T^{\prime}-N\left(T^{\prime}\right)\right)$. Since $B-T^{\prime}-N\left(T^{\prime}\right)=X-T^{\prime}-N\left(T^{\prime}\right)$, we have $\alpha(B) \geq$ $\alpha\left(B \cap T^{\prime}\right)+\alpha(B)-k+1$, i.e. $\alpha\left(B \cap T^{\prime}\right)<k$.

But $\left|I \cap T^{\prime}\right|=k$ because the algorithm added at least $k$ vertices of $I_{k}=I$ to form $T_{k}=T^{\prime}$. Since $I \subseteq V(B)$, we have $\alpha\left(B \cap T^{\prime}\right) \geq k$, a contradiction.

\section{The analysis of the $2 \alpha(G)-\log _{\tau}(\tau \alpha(G) / 2)$ algorithm}

Definition. Let $f:\{0\} \cup \mathbb{R}^{\geq 1} \rightarrow \mathbb{R}, \tau \in \mathbb{R}$ satisfy the following properties:

P1: $f(0)=0$,

$\mathrm{P} 2: f(4 \sqrt{2}) \leq 1$,

P3: If $1 \leq x \in \mathbb{R}$, then $f(\tau x) \leq 1+f(x)$,

P4: If $1 \leq x, y \in \mathbb{R}$, then $f(2 \sqrt{2} x+2 \sqrt{2} y) \leq f(x)+f(y)$,

P5: If $0 \leq x \leq y \in \mathbb{Z}$, then $f(y) \leq f(x)+y-x$,

P6: If $1 \leq x \leq y \in \mathbb{Z}$ and $1 \leq r \in \mathbb{R}$, then $f(r y) \leq f(r x)+y-x$,

P7: $f$ is non-decreasing so by property $\mathrm{P} 4$, if $x_{1}, \ldots, x_{k} \in \mathbb{R}$ with $x_{i} \geq 1$, then $f\left(\sum_{i} x_{i}\right) \leq \sum_{i} f\left(x_{i}\right)$,

P8: If $1 \leq x \in \mathbb{Z}$, then either $\lceil\sqrt{2}(x-1)\rceil \geq \frac{\tau}{\tau-\sqrt{2}} x$ or $f(2 \sqrt{2} x) \leq f(\tau)$.

P9: If $2 \leq x \in \mathbb{Z}$, then $f(2 \sqrt{2} x) \leq 1+f(x-\lceil(x+1) / 2\rceil)$,

P10: If $2 \leq x \in \mathbb{Z}$, then $\sqrt{2} x \leq \tau\lceil(x-1) / 2\rceil$,

P11: If $2 \leq x \in \mathbb{Z}$, then $f(\sqrt{2} x) \leq 2\lceil(x-1) / 2\rceil$.

We can pick $f(x)=\max \left\{\left\lceil\log _{\tau}(\tau x /(4 \sqrt{2}))\right\rceil, 0\right\}$ for $x>0$ and $f(0)=0$, where $\tau=2 \sqrt{2} /(\sqrt{2}-1) \approx 6.83$.

The goal of this section is to prove the following which implies our main result: 
Theorem 14. The algorithm in Section 2.3 produces a complete minor of size $\lceil n /(2 \alpha(G)-f(2 \sqrt{2} \alpha(G)))\rceil$.

To prove Theorem 14 we use Theorems 3 and 5, so we need to prove that the algorithm uses acceptable operations and give an upper bound for the weight of an independent set.

Notation. Let $\mathcal{F}$ be the partition after the algorithm terminates, and let $G_{b}$ be the spanning subgraph of blue edges after the algorithm terminates. Let $\mathcal{F}_{C}$ be the family before Step $C$ begins, and define $A_{C}=V(C)-\cup_{R \in \mathcal{F}_{C}} R$. If $\alpha(C)>1$, define $\mathcal{F}_{C}^{i}$ to be the family right before Substep $i$ of Step $C$, define $\mathcal{F}_{C}^{5}$ to be the family after all substeps of Step $C$ are completed, and let $A_{C}^{i}=V(C)-\cup_{R \in \mathcal{F}_{C}^{i}} R$. For $T \in \mathcal{F}$ and $1 \leq i \leq 5$, define

$$
T^{C, i}= \begin{cases}S & S \in \mathcal{F}_{C}^{i} \text { and } S \subseteq T \text { if there exists such an } \mathrm{S} \\ \emptyset & \text { otherwise. }\end{cases}
$$

In other words, $T^{C, i}$ is the set in $\mathcal{F}_{C}^{i}$ that gets extended to $T$ during the rest of the algorithm.

Define $\mathcal{H}_{0}(C), \mathcal{H}_{1}(C)$, and $\mathcal{H}_{2}(C)$ to be the partition chosen in Substep 1 of Step $C$.

Lemma 15. Let $T^{C, 1} \in \mathcal{H}_{1}(C) \cup \mathcal{H}_{2}(C)$ such that $T^{C, 1}$ was extended during Substep 2 or Substep 3 of Step $C$. This extension satisfies all the conditions of an extension.

Proof. Let $A$ be the set of vertices not in a set of $\mathcal{F}$ right before the extension, $D$ the component of $G[A]$ which $T^{C, 1}$ is extended into and $G_{b}^{\prime}$ the spanning subgraph of blue edges at the time of the extension. Since $T^{C, 1} \notin \mathcal{H}_{0}(C)$ there are no red edges between $T^{C, 1}$ and $C$ so no red edges between $T^{C, 1}$ and $D$. Since each red edge at the time of extension has at least one endpoint in $\mathcal{F}_{C}$, we have $G[V(D)]=G G_{b}^{\prime}[V(D)]$. Since $D$ is a component of $G[A]$, we have that $G_{b}^{\prime}[V(D)]$ is connected. Since we are extending $T^{C, 1}$ we must have $\alpha\left(D-N\left(T^{C, 1}\right)\right)<\alpha(D)$ implying there exist edges between $T^{C, 1}$ and $D$. These edges must be blue so $G_{b}^{\prime}\left[V(D) \cup T^{C, 1}\right]$ is connected. Finally, we extend by $\alpha\left(D-N\left(T^{C, 1}\right)\right)-b+1$ which is smaller than $\alpha\left(D-N\left(T^{C, 1}\right)\right)$ since $b \geq 1$.

Lemma 16. $\mathcal{F}$ is formed by acceptable operations.

Proof. Consider Step $C$. If $\alpha(C)=1$, we break $C$ by 1 . This breaking is acceptable because for each $T \in \mathcal{F}_{C}$ we either have $\alpha(C-N(T))=0<1$ or $\alpha(C-N(T))=\alpha(C)$. So assume $\alpha(C) \geq 2$.

The coloring in Substep 1 can be viewed as breaking $V(C)$ by $\alpha(C)+1$. For $T \in \mathcal{F}$, in Step (a) of breaking $V(C)$ by $\alpha(C)+1$ we colors all edges red between $T$ and $D$, where $D$ is a component of $C$ (i.e. $D=C$ ) with $\alpha(D-N(T))=\alpha(D)$. Since we are breaking by $\alpha(C)+1$, Step (b) of the breaking does not produce any new sets. This breaking is acceptable since for each $T \in \mathcal{F}, \alpha(C-N(T)) \leq \alpha(C)<\alpha(C)+1$.

Extensions are always acceptable, so if Step $C$ does not continue to Substep 3 after Substep 2 then Step $C$ uses acceptable operations. So assume that Step $C$ continues to Substep 3 and then consider the breaking in Substep 4. Since $b=\lceil(\alpha(C)+1) / 2\rceil$, for any component $D$ of $G\left[A_{C}^{4}\right]$ we have $\alpha(D) \leq \alpha(C) \leq 2 b$. So consider some $T^{C, 4} \in \mathcal{F}_{C}^{4}$ and some component $D$ of $G\left[A_{C}^{4}\right]$. If $T^{C, 1} \in \mathcal{H}_{0}(C)$, then $T^{C, 1}=T^{C, 4}$ and $\alpha\left(D-N\left(T^{C, 4}\right)\right) \leq \alpha\left(C-N\left(T^{C, 4}\right)\right)<b$ or all edges between $T^{C, 4}$ and $D$ are colored red.

If $T^{C, 1} \in \mathcal{H}_{1}(C)$, then we considered extending $T^{C, 1}$ in Substep 2. If we extended $T^{C, 1}$ then by Lemmal 7 we must have $\alpha\left(D-N\left(T^{C, 4}\right)\right)<b$. So assume $T^{C, 1}=T^{C, 4}$ and that $b \leq \alpha\left(D-N\left(T^{C, 1}\right)\right)$, and let $D^{\prime}$ be the component of $G\left[A_{C}^{3}\right]$ which contains $D$. Then $b \leq \alpha\left(D^{\prime}-N\left(T^{C, 1}\right)\right)$ and since we continued to Substep 3 we must have $\alpha\left(D^{\prime}-N\left(T^{C, 1}\right)\right)<\alpha\left(D^{\prime}\right)$. In this case we should have continued Substep 2 with the pair $T^{C, 1}, D^{\prime}$. Thus we must have $\alpha\left(D-N\left(T^{C, 1}\right)\right)<b$.

If $T^{C, 1} \in \mathcal{H}_{2}(C)$ then we considered extending $T^{C, 1}$ in Substep 3. If we extended $T^{C, 1}$ then by Lemma 7 we must have $\alpha\left(D-N\left(T^{C, 4}\right)\right)<b$. So assume $T^{C, 1}=T^{C, 4}$. If $b \leq \alpha\left(D-N\left(T^{C, 4}\right)\right)<\alpha(D)$, then we should have continued Substep 3 with the pair $T^{C, 1}, D$. Thus either $\alpha\left(D-N\left(T^{C, 4}\right)\right)<b$ or $\alpha\left(D-N\left(T^{C, 4}\right)\right)=\alpha(D)$, showing that the breaking in Substep 4 is acceptable. 


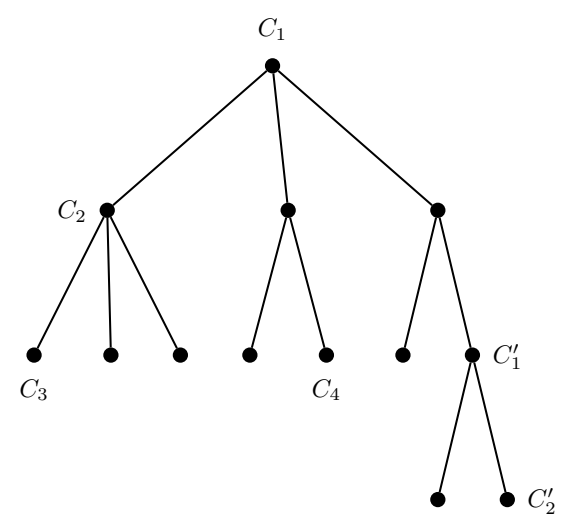

Figure 2: Containment tree of the steps run by the algorithm.

We now need to bound the maximum weight of an independent set. Define the set of independent subfamilies of $\mathcal{F}$ in $G_{b}$ by

$$
\operatorname{IND}_{G_{b}}(\mathcal{F})=\left\{\mathcal{I} \subseteq \mathcal{F}: \mathcal{I} \text { is a pairwise non-touching subfamily in } G_{b}\right\} .
$$

Independent subfamilies of $\mathcal{F}$ correspond to independent sets in $H$.

Using the weight function which measures a set with its size, the total weight is $|V(G)|$. Then the total weight of $\mathcal{I} \in \operatorname{IND}_{G_{b}}(\mathcal{F})$ is $\sum_{T \in \mathcal{I}}|T|$. Using Lemma 6. we know that the weight of $\mathcal{I}$ is at most $2 \sum_{T \in \mathcal{I}} \operatorname{ext}(T)-|\mathcal{I}|$. We will give an upper bound of $2 \alpha(G)-f(2 \sqrt{2} \alpha(G))$ on the weight. To do this, we prove the following inequality

$$
f(2 \sqrt{2} \alpha(G)) \leq|\mathcal{I}|+2 \alpha(G)-2 \sum_{T \in \mathcal{I}} \operatorname{ext}(T) .
$$

Note that when we analyzed the Section 2.2 algorithm, we showed that either $|\mathcal{I}|$ is at least 2 or that $\alpha(G)-\sum_{T \in \mathcal{I}} \operatorname{ext}(T)$ is at least 1. That is, we showed that in order for the total amount of extensions of sets in $\mathcal{I}$ to be $\alpha(G)$ we need more than one set.

Consider Figure 2, where the vertices of the tree are the steps run by the algorithm. Each step of the algorithm corresponds to a component, and the tree is the containment tree of these components. Let $\mathcal{I} \in \operatorname{IND}_{G_{b}}(\mathcal{F})$, with $T, S \in \mathcal{I}$. Say that $T$ is born in the step labeled $C_{1}$ in the figure, and is extended during the steps labeled $C_{2}, C_{3}$, and $C_{4}$. Assume that $S$ is born in the step labeled $C_{1}^{\prime}$ and is extended in the step labeled $C_{2}^{\prime}$. We would like to prove by induction on the steps of the algorithm that

$$
|\{Q \in \mathcal{I}: Q \subseteq V(C)\}|+2 \alpha(C)-2 \sum\{\operatorname{ext}(Q): Q \in \mathcal{I}, Q \subseteq V(C)\}
$$

is large. We are unable to prove this directly because when the induction reaches Step $C_{1}$ we must include $\operatorname{ext}(T)$ into the sum for the first time because Step $C_{1}$ is the first step where $T$ is completely contained inside the component for the step. Instead, we would like our inductive bound for Step $C_{2}$ to include the amount of extensions of $T$ carried out in steps $C_{2}$ and $C_{3}$ which is only part of $\operatorname{ext}(T)$, so that when we reach Step $C_{1}$ the inductive bounds for the smaller components contained inside $C_{1}$ already include most of the value $\operatorname{ext}(T)$. So we define a notion of the gap between $\alpha(C)$ and $\sum\{\operatorname{ext}(Q): Q \in \mathcal{I}, Q \subseteq V(C)\}$ which allows us to include only the amount extensions of $T$ into some subset of $V(C)$. Note that since $\mathcal{F}$ is a partial simplicial elimination ordering, we can have at most one set $T$ which has part of its extensions inside $C$ and part outside $C$. Define for any $T \in \mathcal{I}$

$$
\begin{aligned}
\operatorname{ext}(C, T) & =\text { the total amount of extensions of } T \text { into } X \text { where } X \subseteq V(C), \\
\operatorname{gap}(C, \mathcal{I}, T) & =\alpha\left(C-N\left(T^{C, 1}\right)\right)-\operatorname{ext}(C, T)-\sum\{\operatorname{ext}(Q): Q \in \mathcal{I}, Q \neq T \text { and } Q \cap V(C) \neq \emptyset\} .
\end{aligned}
$$


Note that if $T \cap V(C) \neq \emptyset$ then for each $Q$ in the sum we must have $Q \subseteq V(C)$ because $\mathcal{F}$ is a partial simplicial elimination ordering. In addition, define

$$
\begin{aligned}
\operatorname{ext}(C, \emptyset) & =0, \\
\operatorname{gap}(C, \mathcal{I}, \emptyset) & =\alpha(C)-\sum_{\substack{Q \in \mathcal{I} \\
Q \cap V(C) \neq \emptyset}} \operatorname{ext}(Q) .
\end{aligned}
$$

In the next lemma, we show that $|\mathcal{I}|+2 \operatorname{gap}(C, \mathcal{I}, T)$ is large by induction on the number of steps carried out by the algorithm. For comparison with the Section 2.2 algorithm, Claim 8 proves $0 \leq \operatorname{gap}(G, \mathcal{I}, \emptyset)$.

Lemma 17. Consider any component $C$ chosen as a Step during the algorithm. Let $\mathcal{I}_{0} \in \operatorname{IND}_{G_{b}}(\mathcal{F})$, and let $\mathcal{I}=\left\{Q \in \mathcal{I}_{0}: Q \cap V(C) \neq \emptyset\right\}$.

(i) If $\alpha(C)>1$ and there exists $T \in \mathcal{I}$ with $T^{C, 1} \neq \emptyset$ and $T^{C, 1} \in \mathcal{H}_{1}(C)$ then

$$
f\left(\alpha\left(C-N\left(T^{C, 1}\right)\right)\right) \leq|\mathcal{I}|+2 \operatorname{gap}(C, \mathcal{I}, T)-1 .
$$

(ii) If $\alpha(C)>1$ and there exists $T \in \mathcal{I}$ with $T^{C, 1} \neq \emptyset$ and $T^{C, 1} \in \mathcal{H}_{2}(C)$ then

$$
f\left(\sqrt{2} \alpha\left(C-N\left(T^{C, 1}\right)\right)\right) \leq|\mathcal{I}|+2 \operatorname{gap}(C, \mathcal{I}, T)-1 .
$$

(iii) Otherwise,

$$
f(2 \sqrt{2} \alpha(C)) \leq|\mathcal{I}|+2 \operatorname{gap}(C, \mathcal{I}, \emptyset) .
$$

Using Lemma 17, we can prove Theorem 14 .

Proof of Theorem 14. Let $H$ be the graph formed from $G_{b}$ by contracting each set of $\mathcal{F}$. Define $g$ to be the weight function on $V(H)$ which assigns to each $v \in V(H)$ the size of the set of $\mathcal{F}$ which contracted to $v$. By Lemma 16 and Theorem $[5$ is a perfect graph, so by Theorem 3 we just need to show that $\alpha_{g}(H) \leq 2 \alpha(G)-f(2 \sqrt{2} \alpha(G))$.

Let $C_{1}, \ldots, C_{k}$ be the components of $G$. Let $I$ be any independent set in $H$, which corresponds to a subfamily $\mathcal{I} \in \operatorname{IND}_{G_{b}}(\mathcal{F})$. Then define $\mathcal{I}_{i}=\left\{T \in \mathcal{I}: T \cap V\left(C_{i}\right) \neq \emptyset\right\}$. Since $C_{1}, \ldots, C_{k}$ are components of $G$ we have $\mathcal{I}_{i}=\left\{T \in \mathcal{I}: T \subseteq V\left(C_{i}\right)\right\}$.

For each $T \in \mathcal{I}_{i}$, we have $T \subseteq V\left(C_{i}\right)$ which implies $T^{C_{i}, 1}=\emptyset$. Thus we apply the bound in case (iii) of Lemma 17 for each component $C_{i}$ to obtain

$$
\sum_{1 \leq i \leq k} f\left(2 \sqrt{2} \alpha\left(C_{i}\right)\right) \leq \sum_{1 \leq i \leq k}\left|\mathcal{I}_{i}\right|+2 \sum_{1 \leq i \leq k} \operatorname{gap}\left(C_{i}, \mathcal{I}_{i}, \emptyset\right) .
$$

Expanding the definition of $\operatorname{gap}\left(C_{i}, \mathcal{I}_{i}, \emptyset\right)$ in (1) gives,

$$
\sum_{1 \leq i \leq k} f\left(2 \sqrt{2} \alpha\left(C_{i}\right)\right) \leq|\mathcal{I}|+2 \sum_{1 \leq i \leq k} \alpha\left(C_{i}\right)-2 \sum_{1 \leq i \leq k} \sum_{T \in \mathcal{I}_{i}} \operatorname{ext}(T) .
$$

Since $\sum_{i} \alpha\left(C_{i}\right)=\alpha(G)$ and each $T \in \mathcal{I}$ appears in exactly one $\mathcal{I}_{i}$, (2) simplifies to

$$
\sum_{1 \leq i \leq k} f\left(2 \sqrt{2} \alpha\left(C_{i}\right)\right) \leq|\mathcal{I}|+2 \alpha(G)-2 \sum_{T \in \mathcal{I}} \operatorname{ext}(T) .
$$

Using Lemma 6 to bound $|T|$ and rearranging (3), we have

$$
g(I)=\sum_{T \in \mathcal{I}}|T| \leq 2 \sum_{T \in \mathcal{I}} \operatorname{ext}(T)-|\mathcal{I}| \leq 2 \alpha(G)-\sum_{1 \leq i \leq k} f\left(2 \sqrt{2} \alpha\left(C_{i}\right)\right) .
$$


By property P7,

$$
g(I) \leq 2 \alpha(G)-f\left(2 \sqrt{2} \sum_{1 \leq i \leq k} \alpha\left(C_{i}\right)\right)=2 \alpha(G)-f(2 \sqrt{2} \alpha(G)) .
$$

This says every independent set in $H$ has weight at most $2 \alpha(G)-f(2 \sqrt{2} \alpha(G))$, we have $\alpha_{g}(H) \leq 2 \alpha(G)-$ $f(2 \sqrt{2} \alpha(G))$.

Before proving Lemma 17, we need some lemmas:

Lemma 18. Let $T^{C, 1} \in \mathcal{H}_{1}(C)$ and let $R$ be a set born in Substep 4 of Step $C$. Then $T^{C, 4}$ touches $R$ with blue edges.

Proof. Since $T^{C, 1} \in \mathcal{H}_{1}(C)$, all edges between $T^{C, 1}$ and $C$ are colored blue at the start of Step $C$. We produced an $R$ in Substep 4 so $\alpha(C) \geq 2$, so we consider extending $T^{C, 1}$ in Substep 2. Since we continued to Substep 3 after Substep 2, we have for each component $D$ of $G\left[A_{C}^{3}\right], \alpha\left(D-N\left(T^{C, 3}\right)\right)<b$. Now consider the component $D^{\prime}$ of $G\left[A_{C}^{4}\right]$ which contains $R$. Then there exists a component $D$ of $G\left[A_{C}^{3}\right]$ which contains $D^{\prime}$ so $\alpha\left(D^{\prime}-N\left(T^{C, 4}\right)\right) \leq \alpha\left(D-N\left(T^{C, 4}\right)\right)<b$ so no edges incident to $T^{C, 4}$ are colored red during Substep 4 . Also, since $b \leq \alpha(R)$ by Lemma 6 we must have an edge of $G$ between $T^{C, 4}$ and $R$. This edge is colored blue at the end of the algorithm because no edges incident to $T^{C, 4}$ are colored red during Substep 4 and all future edge colorings only color edges between an existing set in $\mathcal{F}$ and a vertex of $A$.

Lemma 19. Let $T^{C, 1} \in \mathcal{H}_{2}(C)$, and assume that $T^{C, 1}$ was extended in Substep 3 of Step $C$. Let $R$ be a set born during Substep 4 of Step $C$. Then $T^{C, 4}$ touches $R$ with blue edges.

Proof. Since $T^{C, 1} \in \mathcal{H}_{2}(C)$ and we extended $T^{C, 1}$, let $A$ be the subset of vertices of $C$ not yet in a set at the time we extend $T^{C, 1}$, and let $D$ be the component of $G[A]$ where $b \leq \alpha\left(D-N\left(T^{C, 1}\right)\right)<\alpha(D)$. By Lemma $7 \alpha\left(D-T^{C, 4}-N\left(T^{C, 4}\right)\right)<b$. Also, consider any other component $D^{\prime}$ of $G[A]$ besides $D$. Since $\alpha(C) / 2<b<\alpha(D)$, we must have $\alpha\left(D^{\prime}\right)<b$ since $\alpha(D)+\alpha\left(D^{\prime}\right) \leq \alpha(C)$. Thus for all components $D^{\prime}$ of $G\left[A-T^{C, 4}\right]$ we have $\alpha\left(D^{\prime}-N\left(T^{C, 4}\right)\right)<b$. Now $R$ is connected so $R$ must be contained inside some component $D^{\prime \prime}$ of $G\left[A_{C}^{4}\right]$ which is contained inside some component $D^{\prime}$ of $G\left[A-T^{C, 4}\right]$. If $T^{C, 4}$ does not touch $R$ in $G$, then $\alpha\left(D-N\left(T^{C, 4}\right)\right) \geq \alpha(R) \geq b$ which gives a contradiction. If $T^{C, 4}$ touches $R$ with red edges, then we must have colored the edges between $T^{C, 4}$ and $D^{\prime \prime}$ red during Substep 4 so $\alpha\left(D^{\prime \prime}-N\left(T^{C, 4}\right)\right)=\alpha\left(D^{\prime \prime}\right) \geq \alpha(R) \geq b$, again giving a contradiction.

Lemma 20. Let $T^{C, 1} \in \mathcal{H}_{1}(C) \cup \mathcal{H}_{2}(C)$. Then $\alpha\left(C-N\left(T^{C, 1}\right)\right)<\alpha(C)$.

Proof. Assume $T^{C, 1} \in \mathcal{H}_{1}(C) \cup \mathcal{H}_{2}(C)$. If $\alpha\left(C-N\left(T^{C, 1}\right)\right)=\alpha(C)$, then we color all edges between $T$ and $C$ red in Substep 1 of Step $C$. Since all edges are red, $T^{C, 1} \in \mathcal{H}_{0}(C)$ which contradicts $T^{C, 1} \in$ $\mathcal{H}_{1}(C) \cup \mathcal{H}_{2}(C)$.

Proof of Lemma 17. The proof works by induction on $|V(C)|$, where Step $C$ is a step carried out by the algorithm. Fix an $\mathcal{I}_{0} \in \operatorname{IND}_{G_{b}}(\mathcal{F})$ and a component $C$ chosen by the algorithm and consider Step $C$. For the rest of this section, let $D_{1}, \ldots, D_{k}$ be the components of $G\left[A_{C}^{5}\right]$. We can then apply induction into each of the components $D_{i}$ because at some future time in the algorithm $D_{i}$ will be selected as a Step. Let $\mathcal{I}=\left\{T \in \mathcal{I}_{0}: T \cap V(C) \neq \emptyset\right\}$ and $\mathcal{I}_{i}=\left\{T \in \mathcal{I}: T \cap V\left(D_{i}\right) \neq \emptyset\right\}$. We can apply induction into $D_{i}$ with the independent subfamily $\mathcal{I}_{i}$.

If $\alpha(C)=1$, we need to show $f(2 \sqrt{2}) \leq|\mathcal{I}|+2 \operatorname{gap}(C, \mathcal{I}, \emptyset)$. If $\operatorname{gap}(C, \mathcal{I}, T)=0$ then $|\mathcal{I}|=1$. As $f$ is non-decreasing, property $\mathrm{P} 2$ shows $f(2 \sqrt{2}) \leq 1$.

Now assume $\alpha(C)>1$ and consider the possibilities for $T \in \mathcal{I}$ with $T^{C, 1} \neq \emptyset$. We cannot have two sets $T, R \in \mathcal{I}$ with $T^{C, 1} \neq \emptyset$ and $R^{C, 1} \neq \emptyset$, because this would contradict that $\mathcal{F}$ forms a partial simplicial elimination ordering since $T$ and $R$ touch $C$ with blue edges $\left(G_{b}[T]\right.$ is connected and $\left.T \cap V(C) \neq \emptyset\right)$ but all edges between $T$ and $R$ are red. Also, we cannot have two sets $T, R \in \mathcal{I}$ which were born in Substep 4 of Step $C$ because the sets born in Substep 4 are pairwise touching using blue edges. Thus define $T$ to be the set in $\mathcal{I}$ with $T^{C, 1} \neq \emptyset$ if it exists and otherwise define $T=\emptyset$, and define $R$ to be the set in $\mathcal{I}$ which was 
born in Substep 4 of Step $C$, otherwise $R=\emptyset$. Note that $T \neq \emptyset$ implies that $T \in \mathcal{I}$ so that $T \cap V(C) \neq \emptyset$ which implies there are blue edges between $T$ and $C$ which implies $T^{C, 1} \notin \mathcal{H}_{0}(C)$. Thus if $T \neq \emptyset$ we need to prove the inequality in either case (i) or (ii) of Lemma 17. If $T=\emptyset$ we need to prove the inequality in case (iii) of Lemma 17

For each $D_{i}$, at most one of $T$ or $R$ can touch $D_{i}$ using blue edges. (If both touch $D_{i}$ using blue edges, then we contradict the partial simplicial elimination ordering.) Define $Q_{i}$ to be $T$ or $R$ depending on which is contained in $\mathcal{I}_{i}$, and define $Q_{i}=\emptyset$ if neither is in $\mathcal{I}_{i}$. Define

$$
\gamma_{i}= \begin{cases}1 & Q_{i}^{D_{i}, 1} \in \mathcal{H}_{1}\left(D_{i}\right) \\ \sqrt{2} & Q_{i}^{D_{i}, 1} \in \mathcal{H}_{2}\left(D_{i}\right) \\ 2 \sqrt{2} & Q_{i}=\emptyset\end{cases}
$$

\section{Claim 21.}

$$
\sum_{1 \leq i \leq k} f\left(\gamma_{i} \alpha\left(D_{i}-N\left(Q_{i}^{C, 5}\right)\right)\right)-\sum_{1 \leq i \leq k} 2 \operatorname{gap}\left(D_{i}, \mathcal{I}_{i}, Q_{i}\right) \leq|\mathcal{I}-\{T, R\}|
$$

We actually use Claim 21 in the following form:

$$
\sum_{i} f\left(\gamma_{i} \alpha\left(C-N\left(Q_{i}^{C, 5}\right)\right)\right)+2 \operatorname{gap}(C, \mathcal{I}, \emptyset)-2 \sum_{i} \operatorname{gap}\left(D_{i}, \mathcal{I}_{i}, \emptyset\right) \leq|\mathcal{I}-\{T, R\}|+2 \operatorname{gap}(C, \mathcal{I}, \emptyset) .
$$

Proof. Assume we have indexed the components so that for $1 \leq i \leq h_{1}, Q_{i}=T$ and for $h_{1}<i \leq h_{2}, Q_{i}=R$ and for $h_{2}<i \leq k, Q_{i}=\emptyset$. We consider Step $D_{i}$. Then $Q_{i}^{D_{i}, 1}$ is the set of $\mathcal{I}_{i}$ which will touch $D_{i}$ in blue and be considered in the statement of Lemma 17, and $\gamma_{i}$ is the coefficient inside the function $f$. Thus for $1 \leq i \leq h_{2}$, we obtain

$$
f\left(\gamma_{i} \alpha\left(D_{i}-N\left(Q_{i}^{D_{i}, 1}\right)\right)\right) \leq\left|\mathcal{I}_{i}\right|-1+2 \operatorname{gap}\left(D_{i}, \mathcal{I}_{i}, Q_{i}\right) .
$$

Note that $D_{i} \cap N\left(Q_{i}^{D_{i}, 1}\right)=D_{i} \cap N\left(Q_{i}^{C, 5}\right)$ so we have

$$
f\left(\gamma_{i} \alpha\left(D_{i}-N\left(Q_{i}^{C, 5}\right)\right)\right) \leq\left|\mathcal{I}_{i}\right|-1+2 \operatorname{gap}\left(D_{i}, \mathcal{I}_{i}, Q_{i}\right) .
$$

Here, we can think of $\left|\mathcal{I}_{i}\right|-1$ as counting the number of sets in $\mathcal{I}_{i}$ besides $Q_{i}$. For $h_{2}<i \leq k$ we obtain

$$
f\left(\gamma_{i} \alpha\left(D_{i}\right)\right) \leq\left|\mathcal{I}_{i}\right|+2 \operatorname{gap}\left(D_{i}, \mathcal{I}_{i}, \emptyset\right) .
$$

Again $\left|\mathcal{I}_{i}\right|$ is counting the sets of $\mathcal{I}_{i}$ besides $Q_{i}$. Thus

$$
\sum_{i=1}^{h_{2}}\left(\left|\mathcal{I}_{i}\right|-1\right)+\sum_{i=h_{2}+1}^{k}\left|\mathcal{I}_{i}\right|=|\mathcal{I}-\{T, R\}| .
$$

Thus summing the inductive bounds over all $i$ we obtain (for $h_{2}<i \leq k, Q_{i}=\emptyset$ so that $\alpha\left(D_{i}-N\left(Q_{i}^{C, 5}\right)\right)=$ $\left.\alpha\left(D_{i}\right)\right)$

$$
\sum_{1 \leq i \leq k} f\left(\gamma_{i} \alpha\left(D_{i}-N\left(Q_{i}^{C, 5}\right)\right)\right) \leq|\mathcal{I}-\{T, R\}|+\sum_{1 \leq i \leq k} 2 \operatorname{gap}\left(D_{i}, \mathcal{I}_{i}, Q_{i}\right)
$$

We finish the proof of Lemma 17 by showing that the inequality in Claim 21 simplifies in all cases to the inequalities in Lemma 17. For the simplification, we add $\operatorname{gap}(C, \mathcal{I}, T)$ to both sides of Claim 21 and then use 
lower bounds on $\operatorname{gap}(C, \mathcal{I}, T)-\sum_{i} \operatorname{gap}\left(D_{i}, \mathcal{I}_{i}, Q_{i}\right)$ and lower bounds on $\sum_{i} f\left(\gamma_{i} \alpha\left(D_{i}-N\left(Q_{i}^{C, 5}\right)\right)\right)$. Define

$$
\begin{aligned}
\theta & =\operatorname{ext}(C, T)-\sum_{1 \leq i \leq k} \operatorname{ext}\left(D_{i}, T\right)=\text { the amount of extensions of } T \text { during Step } C, \\
\lambda & =\sum_{1 \leq i \leq k} \alpha\left(D_{i}-N\left(Q_{i}^{C, 5}\right)\right), \\
J & =\left\{i: \alpha\left(D_{i}-N\left(Q_{i}^{C, 5}\right)\right)>0\right\} .
\end{aligned}
$$

We claim the following bounds.

Bound 1: If $T=R=\emptyset$, then $f(2 \sqrt{2} \lambda) \leq \sum_{i \in J} f\left(\gamma_{i} \alpha\left(D_{i}-N\left(Q_{i}^{C, 5}\right)\right)\right)$,

Bound 2: If $|J| \geq 2$, then $f(2 \sqrt{2} \lambda) \leq \sum_{i \in J} f\left(\gamma_{i} \alpha\left(D_{i}-N\left(Q_{i}^{C, 5}\right)\right)\right)$,

Bound 3: If $J=\{i\}$, then $f\left(\gamma_{i} \lambda\right)=\sum_{i \in J} f\left(\gamma_{i} \alpha\left(D_{i}-N\left(Q_{i}^{C, 5}\right)\right)\right)$,

Bound 4: $f(\lambda) \leq \sum_{i} f\left(\gamma_{i} \alpha\left(D_{i}-N\left(Q_{i}^{C, 5}\right)\right)\right)$,

Bound 5: If $R=\emptyset$, then $\operatorname{gap}(C, \mathcal{I}, T)-\sum_{i} \operatorname{gap}\left(D_{i}, \mathcal{I}_{i}, Q_{i}\right)=\alpha\left(C-N\left(T^{C, 1}\right)\right)-\theta-\lambda$,

Bound 6: If $R \neq \emptyset$, then $\operatorname{gap}(C, \mathcal{I}, T)-\sum_{i} \operatorname{gap}\left(D_{i}, \mathcal{I}_{i}, Q_{i}\right)=\alpha\left(C-N\left(T^{C, 1}\right)\right)-b-\lambda$.

We now justify these bounds. For Bound $1, T=R=\emptyset$ implies that $Q_{i}=\emptyset$ and $\gamma_{i}=2 \sqrt{2}$ for all $i$ so the inequality follows by property $\mathrm{P} 7$. Bound 2 follows from property $\mathrm{P} 4$ since $|J| \geq 2$. Bound 3 is an equality by definition of $\lambda$. For Bound 4, property P7 and $\gamma_{i} \geq 1$ imply

$$
f(\lambda) \leq \sum_{1 \leq i \leq k} f\left(\alpha\left(D_{i}-N\left(Q_{i}^{C, 5}\right)\right)\right) \leq \sum_{1 \leq i \leq k} f\left(\gamma_{i} \alpha\left(D_{i}-N\left(Q_{i}^{C, 5}\right)\right)\right) .
$$

Now we justify Bound 5 ; assume $R=\emptyset$. First, using the definition of $Q_{i}$ we have

$$
\sum_{1 \leq i \leq k} \operatorname{ext}\left(D_{i}, Q_{i}\right)=\sum_{1 \leq i \leq h_{1}} \operatorname{ext}\left(D_{i}, T\right)+\sum_{h_{1}<i \leq k} \operatorname{ext}\left(D_{i}, \emptyset\right)=\sum_{1 \leq i \leq h_{1}} \operatorname{ext}\left(D_{i}, T\right) .
$$

For $h_{1}<i \leq k$, we have $Q_{i}=\emptyset$ implying that $T \cap V\left(D_{i}\right)=\emptyset$ which $\operatorname{implies} \operatorname{ext}\left(D_{i}, T\right)=0$. Thus the equality in (5) expands to

$$
\sum_{1 \leq i \leq k} \operatorname{ext}\left(D_{i}, Q_{i}\right)=\sum_{1 \leq i \leq k} \operatorname{ext}\left(D_{i}, T\right)
$$

Then expanding the definition of gap,

$$
\begin{aligned}
\sum_{1 \leq i \leq k} \operatorname{gap}\left(D_{i}, \mathcal{I}_{i}, Q_{i}\right) & =\sum_{1 \leq i \leq k} \alpha\left(D_{i}-N\left(Q_{i}^{C, 5}\right)\right)-\sum_{1 \leq i \leq k} \operatorname{ext}\left(D_{i}, Q_{i}\right)-\sum_{1 \leq i \leq k} \sum_{\substack{W \in \mathcal{I}_{i} \\
W \neq Q_{i}}} \operatorname{ext}(W) \\
& =\lambda-\sum_{1 \leq i \leq k} \operatorname{ext}\left(D_{i}, T\right)-\sum_{W \in \mathcal{I}, W \neq T} \operatorname{ext}(W) .
\end{aligned}
$$

Then expanding the definition of $\operatorname{gap}(C, \mathcal{I}, T)$ and combining with the equality in (7) gives

$$
\begin{aligned}
\operatorname{gap}(C, \mathcal{I}, T)-\sum_{1 \leq i \leq k} \operatorname{gap}\left(D_{i}, \mathcal{I}_{i}, Q_{i}\right) & =\alpha\left(C-N\left(T^{C, 1}\right)\right)-\operatorname{ext}(T, C)-\sum_{\substack{W \in \mathcal{I} \\
W \neq T}} \operatorname{ext}(W) \\
& -\sum_{1 \leq i \leq k} \operatorname{gap}\left(D_{i}, \mathcal{I}_{i}, Q_{i}\right) \\
& =\alpha\left(C-N\left(T^{C, 1}\right)\right)-\operatorname{ext}(T, C)-\lambda+\sum_{1 \leq i \leq k} \operatorname{ext}\left(D_{i}, T\right) \\
& =\alpha\left(C-N\left(T^{C, 1}\right)\right)-\lambda-\theta .
\end{aligned}
$$


This completes the proof of Bound 5 .

Finally, consider Bound 6 and assume $R \neq \emptyset$. Using the definition of $Q_{i}$ we have

$$
\sum_{1 \leq i \leq k} \operatorname{ext}\left(D_{i}, Q_{i}\right)=\sum_{1 \leq i \leq h_{1}} \operatorname{ext}\left(D_{i}, T\right)+\sum_{h_{1}<i \leq h_{2}} \operatorname{ext}\left(D_{i}, R\right)
$$

By Lemma 18 and Lemma 19 we did not extend $T$ during Step $C$ so that

$$
\sum_{1 \leq i \leq h_{1}} \operatorname{ext}\left(D_{i}, T\right)=\operatorname{ext}(T, C)
$$

Also, if we have an index $i$ with $Q_{i} \neq R$, this implies $R \cap V\left(D_{i}\right)=\emptyset \operatorname{so} \operatorname{ext}\left(D_{i}, R\right)=0$. Combining (8) and (9) gives

$$
\sum_{1 \leq i \leq k} \operatorname{ext}\left(D_{i}, Q_{i}\right)=\operatorname{ext}(C, T)+\sum_{1 \leq i \leq k} \operatorname{ext}\left(D_{i}, R\right)
$$

Then expanding the definition of gap, we have

$$
\begin{aligned}
\sum_{1 \leq i \leq k} \operatorname{gap}\left(D_{i}, \mathcal{I}_{i}, Q_{i}\right) & =\sum_{1 \leq i \leq k} \alpha\left(D_{i}-N\left(Q_{i}^{C, 5}\right)\right)-\sum_{1 \leq i \leq k} \operatorname{ext}\left(D_{i}, Q_{i}\right)-\sum_{1 \leq i \leq k} \sum_{W \in \mathcal{I}_{i}} \operatorname{ext}(W) \\
& =\lambda-\operatorname{ext}(C, T)-\sum_{1 \leq i \leq k} \operatorname{ext}\left(D_{i}, R\right)-\sum_{W \in \mathcal{I}, W \neq T, W \neq R} \operatorname{ext}(W) \\
& =\lambda-\operatorname{ext}(C, T)+\operatorname{ext}(R)-\sum_{1 \leq i \leq k} \operatorname{ext}\left(D_{i}, R\right)-\sum_{W \in \mathcal{I}, W \neq T} \operatorname{ext}(W) \\
& =\lambda-\operatorname{ext}(C, T)+b-\sum_{W \in \mathcal{I}, W \neq T} \operatorname{ext}(W) .
\end{aligned}
$$

The last inequality holds because $\operatorname{ext}(R)-\sum_{i} \operatorname{ext}\left(D_{i}, R\right)$ is one plus the number of extensions of $R$ during Substep 4 of Step $C$ which is $b$. Then expanding the definition of $\operatorname{gap}(C, \mathcal{I}, T)$ and combining with the equality in (10) gives

$$
\begin{aligned}
\operatorname{gap}(C, \mathcal{I}, T)-\sum_{1 \leq i \leq k} \operatorname{gap}\left(D_{i}, \mathcal{I}_{i}, Q_{i}\right) & =\alpha\left(C-N\left(T^{C, 1}\right)\right)-\operatorname{ext}(C, T)-\sum_{\substack{W \in \mathcal{I} \\
W \neq T}} \operatorname{ext}(W) \\
& -\sum_{\substack{1 \leq i \leq k\\
}} \operatorname{gap}\left(D_{i}, \mathcal{I}_{i}, Q_{i}\right) \\
& =\alpha\left(C-N\left(T^{C, 1}\right)\right)-\lambda-b .
\end{aligned}
$$

This finishes the proof of all the bounds.

We now just need to show that in all the different cases, the inequality in Claim 21$]$ simplifies to the inequalities in Lemma 17

Case 1. $R=T=\emptyset$.

We apply Bounds 1 and 5 to simplify (4)

$$
f(2 \sqrt{2} \lambda)+2 \alpha(C)-2 \lambda \leq|\mathcal{I}|+2 \operatorname{gap}(C, \mathcal{I}, \emptyset) .
$$

Since $\lambda \leq \alpha(C)$ we use property P6 with $r=2 \sqrt{2}$ to obtain

$$
f(2 \sqrt{2} \alpha(C)) \leq f(2 \sqrt{2} \alpha(C))+\alpha(C)-\lambda \leq f(2 \sqrt{2} \lambda)+2 \alpha(C)-2 \lambda .
$$

Combining (11) with (12) proves the inequality in case (iii) of Lemma 17. 
Case 2. $R=\emptyset, T \neq \emptyset, T$ was extended during Step $C$, and $|J| \geq 2$.

We apply Bounds 2 and 5 to simplify (4):

$$
f(2 \sqrt{2} \lambda)+2 \alpha\left(C-N\left(T^{C, 1}\right)\right)-2 \theta-2 \lambda \leq|\mathcal{I}|-1+2 \operatorname{gap}(C, \mathcal{I}, T) .
$$

We have $\alpha\left(C-N\left(T^{C, 1}\right)\right) \geq \theta+\lambda$ since $\lambda=\sum_{i} \alpha\left(D_{i}-N\left(T^{C, 5}\right)\right)$ and $\alpha(T)$ increased by at least $\theta$ during Step $C$ by adding vertices from $C-N\left(T^{C, 1}\right)$. Also, $\lambda \geq 1$ since $J \neq \emptyset$. Thus we can apply property $\mathrm{P} 6$ with $r=2 \sqrt{2}$ to get

$$
\begin{aligned}
f(2 \sqrt{2} \lambda)+2 \alpha\left(C-N\left(T^{C, 1}\right)\right)-2 \theta-2 \lambda & \geq f\left(2 \sqrt{2} \alpha\left(C-N\left(T^{C, 1}\right)\right)-2 \sqrt{2} \theta\right) \\
& \geq f(2 \sqrt{2}(b-1)) .
\end{aligned}
$$

The last inequality holds because $\theta \leq \alpha\left(C-N\left(T^{C, 1}\right)\right)-b+1$ and $f$ is non-decreasing. Now we combine (13) with (14) to obtain

$$
f(2 \sqrt{2}(b-1)) \leq|\mathcal{I}|-1+2 \operatorname{gap}(C, \mathcal{I}, T) .
$$

Then by definition of $b$ we have for $\alpha(C) \geq 2$

$$
2 \sqrt{2}(b-1)=2 \sqrt{2}\left\lceil\frac{\alpha(C)-1}{2}\right\rceil \geq \sqrt{2}(\alpha(C)-1) .
$$

Since we extended $T^{C, 1}$ we have $T^{C, 1} \in \mathcal{H}_{1}(C) \cup \mathcal{H}_{2}(C)$ so by Lemma 20, $\alpha\left(C-N\left(T^{C, 1}\right)\right) \leq \alpha(C)-1$. Then since $f$ is non-decreasing, (15) and (16) simplify to

$$
f\left(\sqrt{2} \alpha\left(C-N\left(T^{C, 1}\right)\right)\right) \leq f(\sqrt{2}(\alpha(C)-1)) \leq|\mathcal{I}|-1+2 \operatorname{gap}(C, \mathcal{I}, T) .
$$

This proves the inequality in case (i) and (ii) of Lemma 17

Case 3. $R=\emptyset, T \neq \emptyset, T$ was extended during Step $C, J=\{i\}$, and we continued to Substep 3.

Then we use Bounds 3 and 5 to simplify (4):

$$
f\left(\gamma_{i} \lambda\right)+2 \alpha\left(C-N\left(T^{C, 1}\right)\right)-2 \lambda-2 \theta \leq|\mathcal{I}|-1+2 \operatorname{gap}(C, \mathcal{I}, T) .
$$

Since $J \neq \emptyset$, we have $\lambda \geq 1$. Also, $\lambda+\theta \leq \alpha\left(C-N\left(T^{C, 1}\right)\right)$ since $\alpha(T)$ increased by at least $\theta$ during Step $C$ and $\lambda=\sum_{i} \alpha\left(C-N\left(T^{C, 5}\right)\right)$. Thus we can apply property $\mathrm{P} 6$ with $r=\gamma_{i}$ to obtain

$$
\begin{aligned}
f\left(\gamma_{i} \lambda\right)+2 \alpha\left(C-N\left(T^{C, 1}\right)\right)-2 \theta-2 \lambda & \geq f\left(\gamma_{i} \alpha\left(C-N\left(T^{C, 1}\right)\right)-\gamma_{i} \theta\right)+\alpha\left(C-N\left(T^{C, 1}\right)\right)-\theta-\lambda \\
& \geq f\left(\gamma_{i}(b-1)\right)+b-1-\lambda .
\end{aligned}
$$

The last inequality holds because $\theta \leq \alpha\left(C-N\left(T^{C, 1}\right)\right)-b+1$ and $f$ is non-decreasing. If $\lambda<b-1$, then using properties P10 and P3 and $\gamma_{i} \geq 1$ we obtain

$$
f\left(\gamma_{i}(b-1)\right)+b-1-\lambda \geq f(b-1)+1 \geq f(\tau(b-1)) \geq f(\sqrt{2} \alpha(C)) .
$$

Since $\alpha\left(C-N\left(T^{C, 1}\right)\right) \leq \alpha(C)$, we combine (17), (18), and (19) to prove the inequality in case (i) and (ii) of Lemma 17

Now assume $\lambda \geq b-1$. Since $|J|=1$ we have $\lambda=\alpha\left(D_{i}-N\left(Q_{i}^{C, 5}\right)\right) \leq \alpha\left(D_{i}\right)$ and since we ran Substep 4 we have $\alpha\left(D_{i}\right) \leq b-1$ so $\lambda=b-1$. We are also forced to have $\alpha\left(D_{i}-N\left(Q_{i}^{C, 5}\right)\right)=\alpha\left(D_{i}\right)$. Assume $Q_{i} \neq \emptyset$. Since $D_{i} \cap N\left(Q_{i}^{C, 5}\right)=D_{i} \cap N\left(Q_{i}^{D_{i}, 1}\right)$ we will color all edges between $Q_{i}^{D_{i}, 1}$ and $D_{i}$ red in Substep 1 of Step $D_{i}$, which implies $Q_{i}^{C, 5} \cap V\left(D_{i}\right)=\emptyset$. This contradicts that $Q_{i} \in \mathcal{I}_{i}$. Thus $Q_{i}=\emptyset$ and so by definition of $\gamma_{i}$, we have $\gamma_{i}=2 \sqrt{2}$. Then we combine (17) with (18) to obtain $(\lambda=b-1)$

$$
f(2 \sqrt{2}(b-1)) \leq|\mathcal{I}|-1+2 \operatorname{gap}(C, \mathcal{I}, T) .
$$


By the inequality in (16) we have $2 \sqrt{2}(b-1) \geq \sqrt{2}(\alpha(C)-1)$. Since $f$ is non-decreasing, (20) simplifies to

$$
f(\sqrt{2}(\alpha(C-1))) \leq|\mathcal{I}|-1+2 \operatorname{gap}(C, \mathcal{I}, T) .
$$

Since $T^{C, 1} \in \mathcal{H}_{1}(C) \cup \mathcal{H}_{2}(C)$ we have by Lemma 20 that $\alpha\left(C-N\left(T^{C, 1}\right)\right)<\alpha(C)$. Thus we have proved the inequality in case (i) and (ii) of Lemma 17.

Case 4. $R=\emptyset, T \neq \emptyset, T$ was extended during Step $C, J=\{i\}$, and we did not continue to Substep 3 after Substep 2.

In this case, we must have $T^{C, 1} \in \mathcal{H}_{1}(C)$ because we did not run Substep 3. We apply Bounds 3 and 5 to simplify (4):

$$
f\left(\gamma_{i} \lambda\right)+2 \alpha\left(C-N\left(T^{C, 1}\right)\right)-2 \lambda-2 \theta \leq|\mathcal{I}|-1+2 \operatorname{gap}(C, \mathcal{I}, T) .
$$

First assume that $\lambda<b-1$. We use $\gamma_{i} \geq 1$ and that $f$ is non-decreasing to simplify (21) to

$$
f(\lambda)+2 \alpha\left(C-N\left(T^{C, 1}\right)\right)-2 \lambda-2 \theta \leq|\mathcal{I}|-1+2 \operatorname{gap}(C, \mathcal{I}, T) .
$$

Because $\alpha(T)$ increased by at least $\theta$ during Step $C$, we have $\lambda+\theta \leq \alpha\left(C-N\left(T^{C, 1}\right)\right)$. We use properties P6 $(J \neq \emptyset$ so $\lambda \geq 1)$, P3, and P10 to obtain

$$
\begin{aligned}
f(\lambda)+2 \alpha\left(C-N\left(T^{C, 1}\right)\right)-2 \lambda-2 \theta & \geq f\left(\alpha\left(C-N\left(T^{C, 1}\right)\right)-\theta\right)+\alpha\left(C-N\left(T^{C, 1}\right)\right)-\lambda-\theta \\
& \geq f(b-1)+b-1-\lambda \\
& \geq f(b-1)+1 \\
& \geq f(\tau(b-1)) \\
& \geq f(\sqrt{2} \alpha(C)) .
\end{aligned}
$$

Since $\alpha\left(C-N\left(T^{C, 1}\right)\right) \leq \alpha(C)$, combining (22) with (23) proves the inequality in both case (i) and (ii) of Lemma 17.

Now assume $\lambda \geq b-1$ so that $\alpha\left(D_{i}-N\left(Q_{i}^{C, 5}\right)\right) \geq b-1$. Since we did not continue to Substep 3 after Substep 2 we must have some component $D_{j}$ and some $S^{C, 1} \in \mathcal{H}_{1}(C)$ with $b \leq \alpha\left(D_{j}\right)=\alpha\left(D_{j}-N\left(S^{C, 1}\right)\right) \leq$ $\sqrt{2}(b-1)$. If $i=j$ then we have $\alpha\left(D_{i}\right) \leq \sqrt{2}(b-1)$ and if $i \neq j$ then $\alpha\left(D_{i}\right) \leq \alpha(C)-\alpha\left(D_{j}\right) \leq \alpha(C)-b \leq$ $\sqrt{2}(b-1)$. Thus

$$
\alpha\left(D_{i}-N\left(Q_{i}^{C, 5}\right)\right) \geq b-1 \geq \frac{1}{\sqrt{2}} \sqrt{2}(b-1) \geq \frac{\alpha\left(D_{i}\right)}{\sqrt{2}} .
$$

Since

$$
\alpha\left(D_{i}\right) \geq 2\left\lceil\frac{\alpha\left(D_{i}\right)-1}{2}\right\rceil
$$

(24) implies that

$$
\alpha\left(D_{i}-N\left(Q_{i}^{D_{i}, 1}\right)\right) \geq \frac{\alpha\left(D_{i}\right)}{\sqrt{2}} \geq \sqrt{2}\left(\left\lceil\frac{\alpha\left(D_{i}\right)+1}{2}\right\rceil-1\right) .
$$

This shows that either $Q_{i}=\emptyset$ or $Q_{i}^{D_{i}, 1} \in \mathcal{H}_{0}\left(D_{i}\right) \cup \mathcal{H}_{2}\left(D_{i}\right)$. If $Q_{i} \neq \emptyset$ and $Q_{i}^{D_{i}, 1} \in \mathcal{H}_{0}\left(D_{i}\right)$ then we must have colored all edges between $Q_{i}^{D_{i}, 1}$ and $D_{i}$ red in Substep 1 of Step $D_{i}$ which contradicts $Q_{i} \cap V\left(D_{i}\right) \neq \emptyset$. Thus either $Q_{i}=\emptyset$ so $\gamma_{i}=2 \sqrt{2}$ or $Q_{i} \neq \emptyset$ and $Q_{i}^{D_{i}, 1} \in \mathcal{H}_{2}\left(D_{i}\right)$ so that $\gamma_{i}=\sqrt{2}$. Then (21) simplifies to

$$
f(\sqrt{2} \lambda)+2 \alpha\left(C-N\left(T^{C, 1}\right)\right)-2 \lambda-2 \theta \leq|\mathcal{I}|-1+2 \operatorname{gap}(C, \mathcal{I}, T) .
$$


Because $\alpha(T)$ increased by at least $\theta$ during Step $C$, we have $\lambda+\theta \leq \alpha\left(C-N\left(T^{C, 1}\right)\right)$. Using property $\mathrm{P} 6$ $(J \neq \emptyset$ so $\lambda \geq 1)$ we obtain

$$
f(\sqrt{2} \lambda)+2 \alpha\left(C-N\left(T^{C, 1}\right)\right)-2 \lambda-2 \theta \geq f\left(\sqrt{2} \alpha\left(C-N\left(T^{C, 1}\right)\right)-\sqrt{2} \theta\right) \geq f(\sqrt{2}(b-1)) .
$$

Combining (25) with (26) gives

$$
f(\sqrt{2}(b-1)) \leq|\mathcal{I}|-1+2 \operatorname{gap}(C, \mathcal{I}, T) .
$$

Since $T^{C, 1} \in \mathcal{H}_{1}(C)$ we have $\sqrt{2}(b-1) \geq \alpha\left(C-N\left(T^{C, 1}\right)\right)$ so we have proved the bound in case (i) of Lemma 17.

Case 5. $R=\emptyset, T \neq \emptyset, T$ was extended during Step $C$, and $J=\emptyset$.

We apply Bounds 4 and 5 to (4) and then use $\lambda=0$ and property P1 to obtain

$$
2 \alpha\left(C-N\left(T^{C, 1}\right)\right)-2 \theta \leq|\mathcal{I}|-1+2 \operatorname{gap}(C, \mathcal{I}, T) .
$$

Then $\theta \leq \alpha\left(C-N\left(T^{C, 1}\right)\right)-b+1$ so

$$
2(b-1) \leq|\mathcal{I}|-1+2 \operatorname{gap}(C, \mathcal{I}, T) .
$$

By property P11, $f(\sqrt{2} \alpha(C)) \leq 2(b-1)$. Since $\alpha\left(C-N\left(T^{C, 1}\right)\right) \leq \alpha(C)$ we have that (27) simplifies to

$$
f\left(\sqrt{2} \alpha\left(C-N\left(T^{C, 1}\right)\right)\right) \leq|\mathcal{I}|-1+2 \operatorname{gap}(C, \mathcal{I}, T) .
$$

This proves the inequality in cases (i) and (ii) in Lemma 17

Case 6. $R=\emptyset, T \neq \emptyset$ and $T^{C, 1}=T^{C, 5}$.

Since $T^{C, 1}=T^{C, 5}$, we have $\theta=0$.

We apply Bounds 4 and 5 to simplify (4):

$$
f(\lambda)+2 \alpha\left(C-N\left(T^{C, 1}\right)\right)-2 \lambda \leq|\mathcal{I}|-1+2 \operatorname{gap}(C, \mathcal{I}, T) .
$$

Since $\lambda \leq \alpha\left(C-N\left(T^{C, 1}\right)\right)$, we use property P5 to obtain

$$
f(\lambda)+2 \alpha\left(C-N\left(T^{C, 1}\right)\right)-2 \lambda \geq f\left(\alpha\left(C-N\left(T^{C, 1}\right)\right)\right) .
$$

If $T^{C, 1} \in \mathcal{H}_{1}(C)$, then (29) and (28) prove the inequality in case (i) of Lemma 17

So assume $T^{C, 1} \in \mathcal{H}_{2}(C)$. If $\lambda<\alpha\left(C-N\left(T^{C, 1}\right)\right)$ we can apply property P5 and P3 to obtain

$$
f(\lambda)+2 \alpha\left(C-N\left(T^{C, 1}\right)\right)-2 \lambda \geq f\left(\alpha\left(C-N\left(T^{C, 1}\right)\right)\right)+1 \geq f\left(\tau \alpha\left(C-N\left(T^{C, 1}\right)\right)\right) .
$$

Because $\tau \geq \sqrt{2}$, we can combine (30) with (28) to prove the inequality in case (ii) of Lemma 17

So assume $\lambda=\alpha\left(C-N\left(T^{C, 1}\right)\right)$. Since $T^{C, 1} \in \mathcal{H}_{2}(C)$ we have $\lambda=\alpha\left(C-N\left(T^{C, 1}\right)\right) \geq \sqrt{2}(b-1)>\alpha(C) / 2$. Then $|J| \geq 2$ since each component of $G\left[A_{C}^{4}\right]$ has independence number at most $\alpha(C) / 2$ and $\lambda>\alpha(C) / 2$. Using $|J| \geq 2$ we can apply Bounds 2 and 5 to Claim 21 to get

$$
f(2 \sqrt{2} \lambda) \leq|\mathcal{I}|-1+2 \operatorname{gap}(C, \mathcal{I}, T) .
$$

Since $\lambda=\alpha\left(C-N\left(T^{C, 1}\right)\right)$, we have proved the bound in case (ii) of Lemma 17 .

Case 7. $R \neq \emptyset$ and $T \neq \emptyset$. 
First, $T^{C, 1} \in \mathcal{H}_{2}(C)$ by Lemma 18 and $T^{C, 4}=T^{C, 1}$ by Lemma 19. Then we apply Bounds 4 and 6 to simplify (4):

$$
f(\lambda)+2 \alpha\left(C-N\left(T^{C, 1}\right)\right)-2 \lambda-2 b \leq|\mathcal{I}|-2+2 \operatorname{gap}(C, \mathcal{I}, T) .
$$

Because $b \leq \alpha\left(R^{C, 5}\right)$ and $Q_{i}$ is $T$ or $R$ we have $\cup_{i}\left(D_{i}-N\left(Q_{i}^{C, 5}\right)\right) \cup R^{C, 5} \subseteq C-N\left(T^{C, 1}\right)$ so that $\lambda+b \leq$ $\alpha\left(C-N\left(T^{C, 1}\right)\right.$ ). (Note that $T^{C, 1} \notin \mathcal{H}_{0}(C)$ so there are no red edges between $T^{C, 1}$ and $R^{C, 5}$.)

Assume $\alpha\left(C-N\left(T^{C, 1}\right)\right)=b$ so that $\lambda=0$. Since $T^{C, 1} \in \mathcal{H}_{2}(C)$ we know $b=\alpha\left(C-N\left(T^{C, 1}\right)\right) \geq \sqrt{2}(b-1)$ so $b$ is one or two so $\alpha\left(C-N\left(T^{C, 1}\right)\right) \leq \alpha(C) \leq 2 b \leq 4$. Thus $f\left(\sqrt{2} \alpha\left(C-N\left(T^{C, 1}\right)\right)\right) \leq f(4 \sqrt{2}) \leq 1$ by property P2. The left side of (31) is zero (using property P1) so adding one to both sides of (31) simplifies to

$$
f\left(\sqrt{2} \alpha\left(C-N\left(T^{C, 1}\right)\right)\right) \leq 1 \leq|\mathcal{I}|-1+2 \operatorname{gap}(C, \mathcal{I}, T)
$$

which is the inequality in case (ii) of Lemma 17.

We now assume $\alpha\left(C-N\left(T^{C, 1}\right)\right)>b$ and we use property P5 to obtain

$$
f(\lambda)+2 \alpha\left(C-N\left(T^{C, 1}\right)\right)-2 \lambda-2 b \geq f\left(\alpha\left(C-N\left(T^{C, 1}\right)\right)-b\right) .
$$

Combining (31) with (32) and adding 1 to both sides we obtain

$$
f\left(\alpha\left(C-N\left(T^{C, 1}\right)\right)-b\right)+1 \leq|\mathcal{I}|-1+2 \operatorname{gap}(C, \mathcal{I}, T) .
$$

Using property P3 this transforms into

$$
f\left(\tau \alpha\left(C-N\left(T^{C, 1}\right)\right)-\tau b\right) \leq|\mathcal{I}|-1+2 \operatorname{gap}(C, \mathcal{I}, T) .
$$

We now apply property P8 with $x=b$. Assume $f(2 \sqrt{2} b) \leq f(\tau)$. Then $\alpha\left(C-N\left(T^{C, 1}\right)\right)-b \geq 1$ and $\alpha\left(C-N\left(T^{C, 1}\right)\right) \leq \alpha(C) \leq 2 b$ imply that

$$
f\left(\sqrt{2} \alpha\left(C-N\left(T^{C, 1}\right)\right)\right) \leq f(2 \sqrt{2} b) \leq f(\tau) \leq f\left(\tau \alpha\left(C-N\left(T^{C, 1}\right)\right)-\tau b\right) .
$$

Combining (34) and (35) we obtain

$$
f\left(\sqrt{2} \alpha\left(C-N\left(T^{C, 1}\right)\right)\right) \leq|\mathcal{I}|-1+2 \operatorname{gap}(C, \mathcal{I}, T),
$$

which is the inequality in case (ii) of Lemma 17.

Therefore, we can assume the other case of property P8 holds, namley that $\lceil\sqrt{2}(b-1)\rceil \geq \frac{\tau}{\tau-\sqrt{2}} b$. Since $T^{C, 1} \in \mathcal{H}_{2}(C)$ we have $\alpha\left(C-N\left(T^{C, 1}\right)\right) \geq \sqrt{2}(b-1)$ so

$$
\alpha\left(C-N\left(T^{C, 1}\right)\right) \geq \frac{\tau}{\tau-\sqrt{2}} b .
$$

Manipulating this inequality, we find

$$
\tau \alpha\left(C-N\left(T^{C, 1}\right)\right)-\tau b \geq \sqrt{2} \alpha\left(C-N\left(T^{C, 1}\right)\right)
$$

so that

$$
f\left(\tau \alpha\left(C-N\left(T^{C, 1}\right)\right)-\tau b\right) \geq f\left(\sqrt{2} \alpha\left(C-N\left(T^{C, 1}\right)\right)\right) .
$$

Combining this with (34) we obtain

$$
f\left(\sqrt{2} \alpha\left(C-N\left(T^{C, 1}\right)\right)\right) \leq|\mathcal{I}|-1+2 \operatorname{gap}(C, \mathcal{I}, T),
$$

which is the inequality in case (ii) of Lemma 17 
Case 8. $R \neq \emptyset$ and $T=\emptyset$.

Using $T=\emptyset$ and $\theta=0$, we apply Bounds 4 and 6 to simplify (4)

$$
f(\lambda)+2 \alpha(C)-2 \lambda-2 b \leq|\mathcal{I}|-1+2 \operatorname{gap}(C, \mathcal{I}, \emptyset) .
$$

Since $\lambda=\sum_{i} \alpha\left(D_{i}-N\left(R^{C, 5}\right)\right)$ and $b \leq \alpha\left(R^{C, 5}\right)$ we have $\lambda+b \leq \alpha(C)$. We use property P5 to obtain

$$
f(\alpha(C)-b) \leq f(\alpha(C)-b)+\alpha(C)-b-\lambda \leq f(\lambda)+2 \alpha(C)-2 \lambda-2 b .
$$

We then combine (36) with (37) and add 1 to both sides to obtain

$$
f(\alpha(C)-b)+1 \leq|\mathcal{I}|+2 \operatorname{gap}(C, \mathcal{I}, T) .
$$

Then property P9 shows $f(2 \sqrt{2} \alpha(C)) \leq f(\alpha(C)-b)+1$ so we have proved the inequality in case (iii) of Lemma 17.

\section{References}

[1] K. Appel and W. Haken, Every planar map is four colorable. I. Discharging, Illinois J. Math. 21 (1977), 429-490.

[2] K. Appel, W. Haken, and J. Koch, Every planar map is four colorable. II. Reducibility, Illinois J. Math. 21 (1977), 491-567.

[3] J. Balogh, J. Lenz, and H. Wu, Complete Minors, Independent Sets, and Chordal Graphs, http://arxiv.org/abs/0907.2421.

[4] C. Berge, Les problèmes de coloration en théorie des graphes, Publ. Inst. Statist. Univ. Paris 9 (1960), 123-160.

[5] M. Chudnovsky and A. Fradkin, An approximate version of Hadwiger's conjecture for claw-free graphs, J. Graph Theory 63 (2010), no. 4, 259-278.

[6] G. Dirac, A property of 4-chromatic graphs and some remarks on critical graphs, J. London Math. Soc. 27 (1952), 85-92.

[7] _ On rigid circuit graphs, Abh. Math. Sem. Univ. Hamburg 25 (1961), 71-76.

[8] P. Duchet and H. Meyniel, On Hadwiger's number and the stability number, Graph theory (Cambridge, 1981), North-Holland, Amsterdam, 1982, pp. 71-73.

[9] J. Fox, Complete minors and independence number, to appear in SIAM J. Discrete Math.

[10] H. Hadwiger, Über eine Klassifikation der Streckenkomplexe, Vierteljschr. Naturforsch. Ges. Zürich 88 (1943), 133-142.

[11] K. Kawarabayashi, M. Plummer, and B. Toft, Improvements of the theorem of Duchet and Meyniel on Hadwiger's conjecture, J. Combin. Theory Ser. B 95 (2005), 152-167.

[12] K. Kawarabayashi and Z. Song, Independence number and clique minors, J. Graph Theory 56 (2007), 219-226.

[13] L. Lovász, Normal hypergraphs and the perfect graph conjecture, Discrete Math. 2 (1972), 253-267.

[14] F. Maffray and H. Meyniel, On a relationship between Hadwiger and stability numbers, Discrete Math. 64 (1987), 39-42. 
[15] M. Plummer, M. Stiebitz, and B. Toft, On a special case of Hadwiger's conjecture, Discuss. Math. Graph Theory 23 (2003), 333-363.

[16] N. Robertson, D. Sanders, P. Seymour, and R. Thomas, The four-colour theorem, J. Combin. Theory Ser. B 70 (1997), 2-44.

[17] N. Robertson, P. Seymour, and R. Thomas, Hadwiger's conjecture for $K_{6}$-free graphs, Combinatorica 13 (1993), 279-361.

[18] B. Toft, A survey of Hadwiger's conjecture, Congr. Numer. 115 (1996), 249-283, Surveys in graph theory (San Francisco, CA, 1995).

[19] K. Wagner, Über eine Eigenschaft der ebenen Komplexe, Math. Ann. 114 (1937), 570-590.

[20] D. Wood, Independent sets in graphs with an excluded clique minor, Discrete Math. Theor. Comput. Sci. 9 (2007), 171-175.

[21] D. R. Woodall, Subcontraction-equivalence and Hadwiger's conjecture, J. Graph Theory 11 (1987), 197-204. 


\section{A The $\alpha(G)=5$ algorithm}

Let $G$ be a graph with $\alpha(G)=5$. At any stage of the algorithm, let $U$ be the set of vertices of $G$ not yet added to any set in $\mathcal{F}$. Initially, $U=|V(G)|$.

- Step 1: Let $\mathcal{F}$ be a maximal family of pairwise touching connected sets, with $\alpha(T) \leq 2$ and $|T| \leq$ $2 \alpha(T)-1$ for each $T \in \mathcal{F}$. We consider such a family with the maximum size, that is the maximum value $|\mathcal{F}|$. Set $U=V(G)-\cup_{T \in \mathcal{F}} T$.

- Step 2: For each $T \in \mathcal{F}$ with $|T|=1$, we extend $T$ into $U$ by 1 if $T$ touches $U$ and $T$ does not dominate $U$. We then repeat Step 2 until we have tried to extend every set.

There are now three cases. Case I is when $G[U]$ has no component with independence number 5 but has a component with independence number 4. Case II is when $G[U]$ has no component with independence number 4 or 5 . Case III is when $G[U]$ is connected and has independence number 5 . We run different steps in the three cases.

Here are the steps in Case I:

- Step I.3: For any $T \in \mathcal{F}$ and any component $C$ of $G[U]$ with $\alpha(C)=4$ and $\alpha(C-N(T))=3$, we extend $T$ into $C$ by 1 . We then update $U$ and continue Step I.3 until no pair $T, C$ satisfies the condition.

- Step I.4: Break $U$ by 3 .

- Step I.5: Break $U$ by 2.

- Step I.6: Break $U$ by 1.

Here are the steps in Case II:

- Step II.3: For any $T \in \mathcal{F}$ and any component $C$ of $G[U]$ with $\alpha(C)=3$ and $\alpha(C-N(T))=2$, we extend $T$ into $C$ by 1 . We then update $U$ and continue Step II.3 until no pair $T, C$ satisfies the condition.

- Step II.4: Break $U$ by 2.

- Step II.5: Break $U$ by 1.

Here are the steps for Case III:

- Step III.3: Break $U$ by 4 .

- Step III.4: For any $T \in \mathcal{F}$ with $\alpha(T)=2$ and any component $C$ of $G[U]$ with $\alpha(C)=3$ and $\alpha(C-N(T))=2$, we extend $T$ into $C$ by 1 . We then update $U$ and continue Step III.4 until no pair $T, C$ satisfies the condition.

- Step III.5: Break $U$ by 2.

- Step III.6: Break $U$ by 1.

We claim that using this algorithm, we can find a complete minor of $G$ of size $\frac{5 n}{38}$.

We set up some notation for the sets in the family at different stages of the algorithm. Let $\mathcal{F}$ be the family at the end of the algorithm, and let $G_{b}$ be the spanning subgraph of blue edges at the end of the algorithm. Let $H$ be the graph obtained from $G_{b}$ by contracting each set in $\mathcal{F}$. For each $T \in \mathcal{F}$ we use $T$ to denote both the set in $V(G)$ and the vertex of $H$ obtained by contracting $T$.

Let

$$
\mathcal{F}(s, a)=\{T: T \text { is a set in the final family, } T \text { was first added during step s, } \operatorname{ext}(T)=a\},
$$


where $s \in\{1, I .3, \ldots, I .6, I I .3, \ldots, I I .5, I I I .3, \ldots, I I I .6\}$.

Define

$$
\mathcal{F}(s)=\cup_{a} \mathcal{F}(s, a) .
$$

Note that we do not include the original sets which were added to the family in step $s$, but include the final configuration of the set which includes the original plus any extensions that were made. Define $\mathcal{F}_{s}$ to be the family right after Step $s$. Let $U_{s}$ be the set of vertices not yet added into any set in $\mathcal{F}$ at the end of Step $s$. Set $n=|V(G)|$ and let $\lambda n$ be the size of the largest complete minor of $G$.

Claim 1. $\mathcal{F}(1)$ is a pairwise touching family in $G_{b}$.

Claim 2. $\mathcal{F}$ is a partial simplicial elimination ordering in $G_{b}$, so $H$ is a chordal graph.

Proof. $\mathcal{F}(1)$ is pairwise touching so trivially is a partial simplicial elimination ordering. Then all breakings are acceptable so that by Theorem $9 \mathcal{F}$ is a partial simplicial elimination ordering. Then we form a simplicial elimination ordering of the vertices of $H$, similarly to the proof of Theorem 5 .

Claim 3. Let $\mathcal{I} \in \operatorname{IND}_{G_{b}}(\mathcal{F})$. Then $\sum_{T \in \mathcal{I}} \operatorname{ext}(T) \leq 5$.

Proof. Can be checked by case analysis.

Claim 4. In the Case III algorithm (even if $U_{2}$ is not connected or $U_{2}$ has independence number less than 5), each $T \in \mathcal{F}(1,3)$ touches every set with extension number at least 2 using blue edges.

Proof. Consider a $T \in \mathcal{F}(1,3)$. Then $T$ touches every set in $\mathcal{F}(1)$ by Claim 1 and by Claim 3 every set in $\mathcal{F}(I I I .3)$. So we only need to show that $T$ touches every set in $\mathcal{F}(I I I .5)$. Since $\operatorname{ext}(T)=3$, we must have extended $T$ in step III.4. By Lemma 7 we know $T$ touches each set in $\mathcal{F}(I I I .5)$ using edges of $G$. It is impossible for these edges to be colored red because $\alpha(C-N(T))$ has been reduced to 1 .

Claim 5. In the Case III algorithm (even if $U_{2}$ is not connected or $U_{2}$ has independence number less than 5), $|\mathcal{F}(1,2)|+|\mathcal{F}(1,3)| \leq(8 \lambda-1) n$.

Proof. Let $f: V(H) \rightarrow \mathbb{Z}^{+}$be defined by

$$
f(T)= \begin{cases}|T|+1 & T \in \mathcal{F}(1,2) \cup \mathcal{F}(1,3), \\ |T| & \text { otherwise. }\end{cases}
$$

Let $\mathcal{I} \in \operatorname{IND}_{G_{b}}(\mathcal{F})$. If $\mathcal{I}$ contains just a single set, the largest extension number of a set is 4 which has 7 vertices so we have $f(\mathcal{I}) \leq 8$. Assume $\mathcal{I}$ has at least two sets, and assume $\mathcal{I}$ does not contain any set in $\mathcal{F}(1,2) \cup \mathcal{F}(1,3)$ Then $\left.f(\mathcal{I}) \leq 2 \sum_{T \in \mathcal{I}} \operatorname{ext}(T)\right)-|\mathcal{I}| \leq 8$. Now assume $\mathcal{I}$ contains some sets in $\mathcal{F}(1,2) \cup \mathcal{F}(1,3)$. Since $\mathcal{F}(1,2) \cup \mathcal{F}(1,3) \subseteq \mathcal{F}(1)$ are all pairwise touching, $\mathcal{I}$ can contain at most one of these sets.

Assume $T \in \mathcal{I} \cap \mathcal{F}(1,2)$. Using Claim B, there are two possibilities. One possibility is $\mathcal{I}=\{T, Q, R\}$ with $Q \in \mathcal{F}(I I I .5)$ and $R \in \mathcal{F}(I I I .6)$. For this $\mathcal{I}$, we have $f(\mathcal{I})=|T|+1+|Q|+|R| \leq 8$. The other possibility is $\mathcal{I}=\{T, P, Q, R\}$ where $P, Q, R \in \mathcal{F}(I I I .6)$. For this $\mathcal{I}$, we have $f(\mathcal{I})=|T|+1+3 \leq 7$. Assume $T \in \mathcal{I} \cap \mathcal{F}(1,3)$. By Claim 4 the only possibility is $\mathcal{I}=\{T, R\}$ with $R \in \mathcal{F}(I I I .6)$. But for this $\mathcal{A}$, $f(\mathcal{I}) \leq|T|+1+|R| \leq 7$.

Thus $f(\mathcal{I}) \leq 8$, so by Theorem 3 we have

$$
\lambda n \geq \frac{f(H)}{8} \geq \frac{n+|\mathcal{F}(1,2) \cup \mathcal{F}(1,3)|}{8} .
$$

Claim 6. In Cases I and II, $|\mathcal{F}(1,2)|+|\mathcal{F}(1,3)| \leq(8 \lambda-1) n$. 
Proof. Consider that instead of running the algorithm with Case I or II, we ran the Case III algorithm. Let $\mathcal{F}^{\prime}$ be the family produced by the Case III algorithm. Then by Claim $5,\left|\mathcal{F}^{\prime}(1,2)\right|+\left|\mathcal{F}^{\prime}(1,3)\right| \leq(8 \lambda-1) n$.

We have $\mathcal{F}(1)=\mathcal{F}^{\prime}(1)$ and also have $\mathcal{F}(1,1)=\mathcal{F}^{\prime}(1,1)$. No possible extension of sets in $\mathcal{F}(1,1)$ or $\mathcal{F}^{\prime}(1,1)$ can happen in steps I.3, II.3, or III.4 because after Step 2, for every $T$ in $\mathcal{F}(1,1)$ or $\mathcal{F}^{\prime}(1,1)$ and each component $C$ of $U_{2}$ either $T$ dominates $C$ or $T$ does not touch $C$.

Claim 7. Assume that the algorithm selected Case I or Case II. Then let $\mathcal{I} \in \operatorname{IND}_{G_{b}}(\mathcal{F})$. Then $\mathcal{I}$ satisfies one of the following conditions.

- $|\mathcal{I}|=1$,

- $|\mathcal{I}| \geq 3$,

- $\mathcal{I}=\{T, R\}$ with $\operatorname{ext}(T)+\operatorname{ext}(R)<5$,

- $\mathcal{I}=\{T, R\}$ with $T \in \mathcal{F}(1,2)$ and $R \in \mathcal{F}(I .4)$ (in Case I),

- $\mathcal{I}=\{T, R\}$ with $T \in \mathcal{F}(1,3)$ and $R \in \mathcal{F}(I .5)$ (in Case I),

- $\mathcal{I}=\{T, R\}$ with $T \in \mathcal{F}(1,3)$ and $R \in \mathcal{F}(I I .4)$ (in Case II).

Proof. Say $\mathcal{I}=\{T, R\}$ with $\operatorname{ext}(T)+\operatorname{ext}(R) \geq 5$. We want to show that we must be in the last three options. Since $\operatorname{ext}(T)+\operatorname{ext}(R) \geq 5$ and the algorithms in Case I or II never produce a set with extension number 4, we must have $\operatorname{ext}(T)=3$ and $\operatorname{ext}(R)=2$ or $\operatorname{ext}(T)=2$ and $\operatorname{ext}(R)=3$. Since $\mathcal{F}(1)$ is pairwise touching in $G_{b}$, at most one of them can be in $\mathcal{F}(1)$. Now consider cases separately. In Case I, say $T \in \mathcal{F}(1,2)$. Then we must have $R \in \mathcal{F}(I .4)$ since $\operatorname{ext}(T)=2$ and $\operatorname{ext}(R)=3$. Consider $T \in \mathcal{F}(1,3)$. Then the only possibility of a set with extension number 2 for $R$ is $\mathcal{F}(I .5)$. In Case II, the only place sets with extension number 3 are created is by extending a set in $\mathcal{F}(1)$. Thus $T \in \mathcal{F}(1,3)$ and the only possibility for $R$ is $\mathcal{F}(I I .4)$.

Claim 8. Assume that the algorithm selected Case I or II. Then $\lambda \geq \frac{2}{15}$.

Proof. Use the following weight function $f: V(H) \rightarrow \mathbb{Q}^{+}$by

$$
f(T)=\left\{\begin{array}{lc}
|T|-1 & T \in \mathcal{F}(1,3), \\
|T| & \text { otherwise. }
\end{array}\right.
$$

Using Claim 7, $\alpha_{f}(H) \leq 7$ so by Theorem 3 we have

$$
n-|\mathcal{F}(1,3)| \leq 7 \lambda n .
$$

Combining with Claim [6 we have

$$
n-(8 \lambda n-n) \leq n-|\mathcal{F}(1,3)| \leq 7 \lambda n
$$

implying the claim.

Claim 9. Assume the algorithm selected Case III. Then every $T \in \mathcal{F}(1,1)$ dominates $U_{2}$ in $G_{b}$.

Proof. Consider $T=\{x\} \in \mathcal{F}(1,1)$. In Step 2 we tried to extend $T$ but failed. Since we are in Case III, $U_{2}$ is connected and $\alpha\left(U_{2}\right)=5$. So because $T$ was not extended in Step 2, we have $T$ dominating $U_{2}$ or $T$ does not touch $U_{2}$ in $G$. If $T$ does not touch $U_{2}$ in $G$, we can form an independent set of size 6 by combining $T$ with an independent set in $U_{2}$ of size 5 . This contradicts $\alpha(G)=5$, so that $T$ dominates $U_{2}$ in $G$. Coloring edges red takes place during a breaking, but only if $\alpha(C-N(T))=\alpha(C)$. Since $T$ dominates $U_{2}$, we always have $\alpha(C-N(T))=0<\alpha(C)$ so no edges incident to $T$ are ever colored red. Thus $T$ dominates $U_{2}$ in $G_{b}$. 
Claim 10. Assume that the algorithm selected Case III. The possible subfamilies in $\operatorname{IND}_{G_{b}}(\mathcal{F})$ are a subset of one of the following cases:

1. One set from $\mathcal{F}(1,1)$,

2. One set from $\mathcal{F}(1,2)$, one set from $\mathcal{F}(I I I .5)$, and one set from $\mathcal{F}(I I I .6)$,

3. One set from $\mathcal{F}(1,2)$ and three sets from $\mathcal{F}(I I I .6)$,

4. One set from $\mathcal{F}(1,3)$ and two sets from $\mathcal{F}(I I I .6)$,

5. One set from $\mathcal{F}(I I I .3)$ and one set from $\mathcal{F}(I I I .6)$,

6. Two sets from $\mathcal{F}(I I I .5)$ and one set from $\mathcal{F}(I I I .6)$,

7. One set from $\mathcal{F}(I I I .5)$ and three sets from $\mathcal{F}(I I I .6)$,

8. Five sets from $\mathcal{F}(I I I .6)$.

Proof. Let $\mathcal{I} \in \operatorname{IND}_{G_{b}}(\mathcal{F})$. Assume $\mathcal{I} \cap \mathcal{F}(1)=\emptyset$. Then using Claim 3 , conditions $5-8$ list all possibilities. So assume $\mathcal{I} \cap \mathcal{F}(1) \neq \emptyset$. By Claim 1, we can include at most one set from $\mathcal{F}(1)$. Say $\mathcal{I} \cap \mathcal{F}(1)=\{T\}$. Consider $T \in \mathcal{I} \cap \mathcal{F}(1,1)$. Then by Claim 9, $T$ dominates $U_{2}$ so $|\mathcal{I}|=1$. Consider $T \in \mathcal{I} \cap \mathcal{F}(1,2)$. Then conditions 2 and 3 cover the two possibilities. Consider $T \in \mathcal{I} \cap \mathcal{F}(1,3)$. Then $T$ touches every set in $\mathcal{F}($ III I.5) by Claim 4 . Thus the maximal non-touching family extending $\{T\}$ is adding two sets from $\mathcal{F}(I I I .6)$.

Claim 11. Assume that the algorithm selected Case III. Then $\lambda \geq \frac{5}{38}$.

Proof. First,

$$
n \leq|\mathcal{F}(1,1)|+3|\mathcal{F}(1,2)|+5|\mathcal{F}(1,3)|+7|\mathcal{F}(I I I .3)|+3|\mathcal{F}(I I I .5)|+|\mathcal{F}(I I I .6)| .
$$

Now define $f: V(H) \rightarrow \mathbb{Z}^{+}$to be:

$$
f(T)= \begin{cases}38 & T \in \mathcal{F}(1,1), \\ 21 & T \in \mathcal{F}(1,2), \\ 32 & T \in \mathcal{F}(1,3), \\ 35 & T \in \mathcal{F}(I I I .3), \\ 14 & T \in \mathcal{F}(I I I .5), \\ 3 & T \in \mathcal{F}(I I I .6) .\end{cases}
$$

Then considering Claim 10, we know $\alpha_{f}(H) \leq 38$. Thus using Theorem 3, we have

$$
38 \lambda n \geq 38|\mathcal{F}(1,1)|+21|\mathcal{F}(1,2)|+32|\mathcal{F}(1,3)|+35|\mathcal{F}(I I I .3)|+14|\mathcal{F}(I I I .5)|+3|\mathcal{F}(I I I .6)|
$$

giving

$$
38 \lambda n \geq 5 n+33|\mathcal{F}(1,1)|+6|\mathcal{F}(1,2)|+7|\mathcal{F}(1,3)|-|\mathcal{F}(I I I .5)|-2|\mathcal{F}(I I I .6)| .
$$

Thus if we can show that

$$
|\mathcal{F}(I I I .5)|+2|\mathcal{F}(I I I .6)| \leq 6|\mathcal{F}(1,2)|+7|\mathcal{F}(1,3)|
$$

we will have $38 \lambda n \geq 5 n$ proving the claim.

Define $\mathcal{F}^{\prime}=\mathcal{F}_{2} \cup \mathcal{F}(I I I .6)$. Define $L$ to be the graph formed by starting with $G_{b}\left[\cup_{T \in \mathcal{F}^{\prime}} T\right]$ and contracting each set of $\mathcal{F}^{\prime}$. Then $\mathcal{F}^{\prime}$ is a partial simplicial elimination ordering, so that $L$ is a chordal graph. 
Define $f: V(L) \rightarrow \mathbb{Z}^{+}$by

$$
f(T)= \begin{cases}5 & T \in \mathcal{F}_{2} \text { and } \operatorname{ext}(T)=1, \\ 2 & T \in \mathcal{F}_{2} \text { and } \operatorname{ext}(T)=2, \\ 1 & T \in \mathcal{F}(I I I .6) .\end{cases}
$$

Consider $\mathcal{I} \in \operatorname{IND}_{L}\left(\mathcal{F}^{\prime}\right)$. Say $T \in \mathcal{I} \cap \mathcal{F}_{2}$. If $\operatorname{ext}(T)=1$ then we did not extend $T$ in Step III.4 so $T \in \mathcal{F}(1,1)$ so by Claim 9 , $T$ dominates $U_{2}$ so $|\mathcal{I}|=1$ so $f(\mathcal{I})=5$. If $\operatorname{ext}(T)=2$ then we could have $\mathcal{I}=\{T, P, Q, R\}$ with $P, Q, R \in \mathcal{F}(I I I .6)$. Then $f(\mathcal{I})=5$. If $\mathcal{I} \cap \mathcal{F}_{2}=\emptyset$ then $\mathcal{I}$ can have at most five sets from $\mathcal{F}(I I I .6)$ (by Lemma 3) so $f(\mathcal{I}) \leq 5$.

$L$ is chordal and $\alpha_{f}(L) \leq 5$ so by Theorem 3. $\omega(L) \geq \frac{f(L)}{5}$. This clique in $L$ is a pairwise touching subfamily of $\mathcal{F}^{\prime}$ with size at least $\frac{f(L)}{5}$. This pairwise touching subfamily is a candidate for the choice of a family in Step 1. By the maximum choice in Step 1, we know

$$
|\mathcal{F}(1)| \geq \frac{f(L)}{5} \geq \frac{2|\mathcal{F}(1)|+3|\mathcal{F}(1,1)|+|\mathcal{F}(I I I .6)|}{5} .
$$

Thus

$$
3|\mathcal{F}(1,2)|+3|\mathcal{F}(1,3)| \geq|\mathcal{F}(I I I .6)| .
$$

Now define $\mathcal{F}^{\prime}=\mathcal{F}(1,1) \cup \mathcal{F}(1,2) \cup \mathcal{F}(I I I .5) \cup \mathcal{F}(I I I .6)$. Define $L=G_{b}\left[\cup_{T \in \mathcal{F}^{\prime}} T\right]$. Again, $\mathcal{F}^{\prime}$ is a partial simplicial elimination ordering in $G_{b}\left[\cup_{T \in \mathcal{F}^{\prime}} T\right]$ so that $L$ is a chordal graph.

We then consider the weight function $f: V(L) \rightarrow \mathbb{Z}^{+}$

$$
f(T)= \begin{cases}5 & T \in \mathcal{F}(1,1), \\ 2 & T \in \mathcal{F}(1,2), \\ 2 & T \in \mathcal{F}(I I I .5), \\ 1 & T \in \mathcal{F}(I I I .6) .\end{cases}
$$

Then using Claim 10, we have $f(\mathcal{I}) \leq 5$ for each $\mathcal{I} \in \operatorname{IND}_{G_{b}}\left(\mathcal{F}^{\prime}\right) . \quad L$ is chordal and $\alpha_{f}(L) \leq 5$ so by Theorem 3 we have a clique in $L$ of size $\frac{f(L)}{5}$. This clique corresponds to a pairwise touching subfamily of $\mathcal{F}^{\prime}$ of size $\frac{f(L)}{5}$. Again, this subfamily is a possibility for the family in Step 1. By the maximum choice in Step 1,

$$
|\mathcal{F}(1)| \geq \frac{5|\mathcal{F}(1,1)|+2|\mathcal{F}(1,2)|+2|\mathcal{F}(I I I .5)|+|\mathcal{F}(I I I .6)|}{5},
$$

implying

$$
3|\mathcal{F}(1,2)|+5|\mathcal{F}(1,3)| \geq 2|\mathcal{F}(I I I .5)|+|\mathcal{F}(I I I .6)| .
$$

Using $1.5(38)+0.5$ (39) we obtain

$$
6|\mathcal{F}(1,2)|+7|\mathcal{F}(1,3)| \geq|\mathcal{F}(I I I .5)|+2|\mathcal{F}(I I I .6)| .
$$

